\title{
State of the art of predictive modeling for real-life applications
}

\section{Andreas Sepp}

Abstract- predictive modeling techniques had recently witnessed significant improvement due the advances in artificial intelligence and machine learning. This research presents a survey on the methods and applications of artificial intelligence and machine learning used in predictive analytics.

Mosavi, A., Golshan, M., Choubin, B., Ziegler, A.D., Sigaroodi, S.K., Zhang, F., Dineva, A.A. Fuzzy clustering and distributed model for streamflow estimation in ungauged watersheds (2021) Scientific Reports, 11 (1), art. no. 8243

Haghighat Shoar, F., Najafi, B., Mosavi, A. Effects of triethylene glycol mono methyl ether (TGME) as a novel oxygenated additive on emission and performance of a dual-fuel diesel engine fueled with natural gas-diesel/biodiesel

(2021) Energy Reports, 7, pp. 1172-1189.

Shadkani, S., Abbaspour, A., Samadianfard, S., Hashemi, S., Mosavi, A., Band, S.S. Comparative study of multilayer perceptron-stochastic gradient descent and gradient boosted trees for predicting daily suspended sediment load: The case study of the Mississippi River, U.S.

(2021) International Journal of Sediment Research, 36 (4), pp. 512-523.

Mahmoudi, M.R., Baleanu, D., Band, S.S., Mosavi, A.

Factor analysis approach to classify COVID-19 datasets in several regions

(2021) Results in Physics, 25, art. no. 104071.

Navabi, D., Javidruzi, M., Hafezi, M.R., Mosavi, A.

The high-performance light transmitting concrete and experimental analysis of using polymethylmethacrylate optical fibers in it

(2021) Journal of Building Engineering, 38, art. no. 102076 .

Azareh, A., Sardooi, E.R., Gholami, H., Mosavi, A., Shahdadi, A., Barkhori, S. Detection and prediction of lake degradation using landscape metrics and remote sensing dataset, (2021) Environmental Science and Pollution Research, 28 (21), pp. 27283-27298.

Kalbasi, R., Jahangiri, M., Mosavi, A., Jalaladdin Hosseini Dehshiri, S., Shahabaddin Hosseini Dehshiri, S., Ebrahimi, S., Al-Sadat Etezadi, Z., Karimipour, A.

Finding the best station in Belgium to use residential-scale solar heating, One-year dynamic simulation with considering all system losses: Economic analysis of using ETSW (2021) Sustainable Energy Technologies and Assessments, 45, art. no. 101097 .

Masoomi, M., Yousefifard, M., Mosavi, A. Efficiency assessment of an amended oscillating water column using openfoam (2021) Sustainability (Switzerland), 13 (10), art. no. 5633 .

Karimimoshaver, M., Eris, B., Aram, F., Mosavi, A. Art in urban spaces (2021) Sustainability (Switzerland), 13 (10), art. no. 5597. 
Awan, H.H., Javed, M.F., Yousaf, A., Aslam, F., Alabduljabbar, H., Mosavi, A. Experimental evaluation of untreated and pretreated crumb rubber used in concrete (2021) Crystals, 11 (5), art. no. 558.

Javed, M.F., Durrani, A.A., Rehman, S.K.U., Aslam, F., Alabduljabbar, H., Mosavi, A. Effect of recycled coarse aggregate and bagasse ash on two-stage concrete (2021) Crystals, 11 (5), art. no. 556.

Karimimoshaver, M., Parsamanesh, M., Aram, F., Mosavi, A. The impact of the city skyline on pleasantness; state of the art and a case study (2021) Heliyon, 7 (5), art. no. e07009.

Peng, Y., Ghahnaviyeh, M.B., Ahamd, M.N., Abdollahi, A., Bagherzadeh, S.A., Azimy, H., Mosavi, A., Karimipour, A. Analysis of the effect of roughness and concentration of Fe304/water nanofluid on the boiling heat transfer using the artificial neural network: An experimental and numerical study (2021) International Journal of Thermal Sciences, 163, art. no. 106863.

Mousavi, S.M., Ghasemi, M., Manshadi, M.D., Mosavi, A. Deep learning for wave energy converter modeling using long short-term memory (2021) Mathematics, 9 (8), art. no. 871 .

Mala, A.A., Sherwani, A.F.H., Younis, K.H., Faraj, R.H., Mosavi, A. Mechanical and fracture parameters of ultra-high performance fiber reinforcement concrete cured via steam and water: Optimization of binder content (2021) Materials, 14 (8), art. no. 2016.

Alaloul, W.S., Altaf, M., Musarat, M.A., Javed, M.F., Mosavi, A. Systematic review of life cycle assessment and life cycle cost analysis for pavement and a case study (2021) Sustainability (Switzerland), 13 (8), art. no. 4377.

Shah, M.I., Abunama, T., Javed, M.F., Bux, F., Aldrees, A., Rehman Tariq, M.A.U., Mosavi, A. Modeling surface water quality using the adaptive neurofuzzy inference system aided by input optimization (2021) Sustainability (Switzerland), 13 (8), art. no. 4576 .

Rafiq, W., Musarat, M.A., Altaf, M., Napiah, M., Sutanto, M.H., Alaloul, W.S., Javed, M.F., Mosavi, A. Life cycle cost analysis comparison of hot mix asphalt and reclaimed asphalt pavement: A case study (2021) Sustainability (Switzerland), 13 (8), art. no. 4411 .

Masoomi, M., Mosavi, A. The one-way fsi method based on rans-fem for the open water test of a marine propeller at the different loading conditions (2021) Journal of Marine Science and Engineering, 9 (4), art. no. 351.

Hosseini, F., Sajadzadeh, H., Aram, F., Mosavi, A. The impact of local green spaces of historically and culturally valuable residential areas on place attachment (2021) Land, 10 (4), art. no. 351.

Moayedi, H., Mosavi, A. Suggesting a stochastic fractal search paradigm in combination with artificial neural network for early prediction of cooling load in residential buildings (2021) Energies, 14 (6), art. no. 1649.

Moayedi, H., Mosavi, A. Synthesizing multi-layer perceptron network with ant lion biogeography-based dragonfly algorithm evolutionary strategy invasive weed and league champion optimization hybrid algorithms in predicting heating load 
in residential buildings (2021) Sustainability (Switzerland), 13 (6), art. no. 3198 .

Lashkar-Ara, B., Kalantari, N., Khozani, Z.S., Mosavi, A. Assessing machine learning versus a mathematical model to estimate the transverse shear stress distribution in a rectangular channel (2021) Mathematics, 9 (6), art. no. 596,

Tavoosi, J., Suratgar, A.A., Menhaj, M.B., Mosavi, A., Mohammadzadeh, A., Ranjbar, E. Modeling renewable energy systems by a self-evolving nonlinear consequent part recurrent type-2 fuzzy system for power prediction (2021) Sustainability (Switzerland), 13 (6), art. no. 3301.

Moayedi, H., Mosavi, A. Double-target based neural networks in predicting energy consumption in residential buildings (2021) Energies, 14 (5), art. no. 1331 .

Mousa, M., Nosratabadi, S., Sagi, J., Mosavi, A. The effect of marketing investment on firm value and systematic risk (2021) Journal of Open Innovation: Technology, Market, and Complexity, 7 (1), art. no. 64, pp. 1-17.

Mosavi, A., Sedeh, S.N., Toghraie, D., Karimipour, A. Analysis of entropy generation of ferrofluid flow in the microchannel with twisted porous ribs: The two-phase investigation with various porous layers

(2021) Powder Technology, 380, pp. 349-357.

Khan, M.A., Zafar, A., Akbar, A., Javed, M.F., Mosavi, A. Application of gene expression programming (GEP) for the prediction of compressive strength of geopolymer concrete (2021) Materials, 14 (5), art. no. 1106, pp. 1-23.

Taghizadeh-Mehrjardi, R., Emadi, M., Cherati, A., Heung, B., Mosavi, A., Scholten, T. Bio-inspired hybridization of artificial neural networks: An application for mapping the spatial distribution of soil texture fractions (2021) Remote Sensing, 13 (5), art. no. 1025, pp. 1-23.

Mosavi, A., Sajedi Hosseini, F., Choubin, B., Taromideh, F., Ghodsi, M., Nazari, B., Dineva, A.A. Susceptibility mapping of groundwater salinity using machine learning models (2021) Environmental Science and Pollution Research, 28 (9), pp. 10804-10817.

Shah, M.I., Amin, M.N., Khan, K., Niazi, M.S.K., Aslam, F., Alyousef, R., Javed, M.F., Mosavi, A. Performance evaluation of soft computing for modeling the strength properties of waste substitute green concrete (2021) Sustainability (Switzerland), 13 (5), art. no. 2867, pp. 1-21.

Sattari, M.T., Apaydin, H., Band, S.S., Mosavi, A., Prasad, R. Comparative analysis of kernel-based versus ANN and deep learning methods in monthly reference evapotranspiration estimation (2021) Hydrology and Earth System Sciences, 25 (2), pp. 603-618.

Moayedi, H., Mosavi, A. An innovative metaheuristic strategy for solar energy management through a neural networks framework (2021) Energies, 14 (4), art. no. 1196.

Moayedi, H., Mosavi, A. Electrical power prediction through a combination of multilayer perceptron with water cycle ant lion and satin bowerbird searching optimizers (2021) Sustainability (Switzerland), 13 (4), art. no. 2336, pp. 120 . 
Shahbazpanahi, S., Tajara, M.K., Faraj, R.H., Mosavi, A. Studying the C-H crystals and mechanical properties of sustainable concrete containing recycled coarse aggregate with used nano-silica (2021) Crystals, 11 (2), art. no. 122.

Mahmoudi, M.R., Baleanu, D., Qasem, S.N., Mosavi, A., S. Band, S. Testing the equality of several independent stationary and non-stationary time series models with fractional Brownian motion errors (2021) Alexandria Engineering Journal, 60 (1), pp. 1767-1775.

Mahmoudi, M.R., Baleanu, D., Qasem, S.N., Mosavi, A., S. Band, S. Fuzzy clustering to classify several time series models with fractional Brownian motion errors(2021) Alexandria Engineering Journal, 60 (1), pp. 1137-1145.

Mahmoudi, M.R., Heydari, M.H., Qasem, S.N., Mosavi, A., Band, S.S. Principal component analysis to study the relations between the spread rates of COVID-19 in high risks countries (2021) Alexandria Engineering Journal, 60 (1), pp. 457-464.

Mosavi, A., Hekmatifar, M., Toghraie, D., Sabetvand, R., Alizadeh, A., Sadeghi, Z., Karimipour, A. Atomic interactions between rock substrate and water-sand mixture with and without graphene nanosheets via molecular dynamics simulation (2021) Journal of Molecular Liquids, 323, art. no. 114610 .

Taghizadeh-Mehrjardi, R., Schmidt, K., Toomanian, N., Heung, B., Behrens, T., Mosavi, A., S. Band, S., Amirian-Chakan, A., Fathabadi, A., Scholten, T. Improving the spatial prediction of soil salinity in arid regions using wavelet transformation and support vector regression models(2021) Geoderma, 383, art. no. 114793 .

Ahmad, J., Tufail, R.F., Aslam, F., Mosavi, A., Alyousef, R., Javed, M.F., Zaid, O., Khan Niazi, M.S. A step towards sustainable self-compacting concrete by using partial substitution of wheat straw ash and bentonite clay instead of cement (2021) Sustainability (Switzerland), 13 (2), art. no. 824, pp. 1-17.

Moayedi, H., Mosavi, A. A water cycle-based error minimization technique in predicting the bearing capacity of shallow foundation (2021) Engineering with Computers.

Mohammadzadeh, A., Castillo, O., Band, S.S., Mosavi, A. A Novel FractionalOrder Multiple-Model Type-3 Fuzzy Control for Nonlinear systems with Unmodeled Dynamics (2021) International Journal of Fuzzy Systems.

Homaei, M.H., Band, S.S., Pescape, A., Mosavi, A. DDSLA-RPL: Dynamic Decision System Based on Learning Automata in the RPL Protocol for Achieving QoS (2021) IEEE Access, 9, art. no. 9411861, pp. 63131-63148.

Baghaee, S., Nosratabadi, S., Aram, F., Mosavi, A. Driving factors behind the social role of retail centers on recreational activities (2021) Cogent Business and Management, 8 (1), art. no. 1905218.

Nosratabadi, S., Ardabili, S., Lakner, Z., Mako, C., Mosavi, A. Prediction of food production using machine learning algorithms of multilayer perceptron and anfis (2021) Agriculture (Switzerland), 11 (5), art. no. 408.

Sadeghiravesh, M.H., Khosravi, H., Abolhasani, A., Ghodsi, M., Mosavi, A. Fuzzy logic model to assess desertification intensity based on vulnerability indices (2021) Acta Polytechnica Hungarica, 18 (3), pp. 7-24. 
Mahmoudi, M.R., Rahmati, M., Mansor, Z., Mosavi, A., Band, S.S. A Statistical Approach to Model the $\mathrm{H}$-Index Based on the Total Number of Citations and the Duration from the Publishing of the First Article (2021) Complexity, 2021 , art. no. 6351836 .

Ghaemi, A., Zhian, T., Pirzadeh, B., Hashemi Monfared, S., Mosavi, A. Reliability-based design and implementation of crow search algorithm for longitudinal dispersion coefficient estimation in rivers (2021) Environmental Science and Pollution Research.

Moosanezhad-Kermani, H., Rezaei, F., Hemmati-Sarapardeh, A., Band, S.S., Mosavi, A. Modeling of carbon dioxide solubility in ionic liquids based on group method of data handling (2021) Engineering Applications of Computational Fluid Mechanics, 15 (1), pp. 23-42.

Mosavi, A., Zarringhalam, M., Toghraie, D., Rahmani, A., Karimipour, A. Boiling of Argon flow in a microchannel by considering the spherical geometry for roughness barriers using molecular dynamics simulation (2021) Journal of Molecular Liquids, 321, art. no. 114462.

Bonakdari, H., Ebtehaj, I., Gharabaghi, B., Sharifi, A., Mosavi, A. Prediction of Discharge Capacity of Labyrinth Weir with Gene Expression Programming (2021) Advances in Intelligent Systems and Computing, 1250 AISC, pp. 202-217.

Band, S.S., Bateni, S.M., Almazroui, M., Sajjadi, S., Chau, K.-W., Mosavi, A. Evaluating the potential of offshore wind energy in the Gulf of Oman using the MENA-CORDEX wind speed data simulations (2021) Engineering Applications of Computational Fluid Mechanics, 15 (1), pp. 613-626.

Karimmaslak, H., Najafi, B., Band, S.S., Ardabili, S., Haghighat-Shoar, F., Mosavi, A. Optimization of performance and emission of compression ignition engine fueled with propylene glycol and biodiesel-diesel blends using artificial intelligence method of ANN-GA-RSM (2021) Engineering Applications of Computational Fluid Mechanics, 15 (1), pp. 413-425.

Sun, X., Bi, Y., Karami, H., Naini, S., Band, S.S., Mosavi, A. Hybrid model of support vector regression and fruitfly optimization algorithm for predicting ski-jump spillway scour geometry (2021) Engineering Applications of Computational Fluid Mechanics, 15 (1), pp. 272-291.

Najafi, B., Haghighatshoar, F., Ardabili, S., S. Band, S., Chau, K.W., Mosavi, A. Effects of low-level hydroxy as a gaseous additive on performance and emission characteristics of a dual fuel diesel engine fueled by diesel/biodiesel blends (2021) Engineering Applications of Computational Fluid Mechanics, 15 (1), pp. 236-250.

Liu, Z., Mohammadzadeh, A., Turabieh, H., Mafarja, M., Band, S.S., Mosavi, A. A new online learned interval type-3 fuzzy control system for solar energy management systems (2021) IEEE Access, 9, art. no. 9314138, pp. 10498-10508.

Mosavi, A., Sajedi Hosseini, F., Choubin, B., Goodarzi, M., Dineva, A.A., Rafiei Sardooi, E. Ensemble Boosting and Bagging Based Machine Learning Models for Groundwater Potential Prediction (2021) Water Resources Management, 35 (1), pp. 23-37. 
Nguyen, Q., Bagherzadeh, S.A., Parsian, A., Akbari, M., Karimipour, A., Mosavi, A. Nonlinear model identification of dissimilar laser joining of S.S 304 and ABS using the Hammerstein-Wiener method

(2021) Optik, 225, art. no. 165649, .

Sun, K., Rajabtabar, M., Samadi, S.Z., Rezaie-Balf, M., Ghaemi, A., Band, S.S., Mosavi, A. An integrated machine learning, noise suppression, and populationbased algorithm to improve total dissolved solids prediction (2021) Engineering Applications of Computational Fluid Mechanics, 15 (1), pp. 251-271.

Mosavi, A., Samadianfard, S., Darbandi, S., Nabipour, N., Qasem, S.N., Salwana, E., S. Band, S. Predicting soil electrical conductivity using multi-layer perceptron integrated with grey wolf optimizer (2021) Journal of Geochemical Exploration, 220, art. no. 106639.

Iqtidar, A., Khan, N.B., Kashif-ur-Rehman, S., Javed, M.F., Aslam, F., Alyousef, R., Alabduljabbar, H., Mosavi, A. Prediction of compressive strength of rice husk ash concrete through different machine learning processes (2021) Crystals, 11 (4), art. no. 352.

Chen, Y., Chen, W., Chandra Pal, S., Saha, A., Chowdhuri, I., Adeli, B., Janizadeh, S., Dineva, A.A., Wang, X., Mosavi, A. Evaluation efficiency of hybrid deep learning algorithms with neural network decision tree and boosting methods for predicting groundwater potential (2021) Geocarto International.

Rahman, A., Islam, M.J., Montieri, A., Nasir, M.K., Reza, M.M., Band, S.S., Pescape, A., Hasan, M., Sookhak, M., Mosavi, A.

SmartBlock-SDN: An Optimized Blockchain-SDN Framework for Resource Management in IoT (2021) IEEE Access, 9, art. no. 9350593, pp. 28361-28376.

Kekha Javan, A.A., Shoeibi, A., Zare, A., Hosseini Izadi, N., Jafari, M., Alizadehsani, R., Moridian, P., Mosavi, A., Acharya, U.R., Nahavandi, S. Design of adaptive-robust controller for multi-state synchronization of chaotic systems with unknown and time-varying delays and its application in secure communication (2021) Sensors (Switzerland), 21 (1), art. no. 254, pp. 1-21.

Mousavi, S.P., Atashrouz, S., Nait Amar, M., Hemmati-Sarapardeh, A., Mohaddespour, A., Mosavi, A. Viscosity of Ionic Liquids: Application of the Eyring's Theory and a Committee Machine Intelligent System (2020) Molecules (Basel, Switzerland), 26 (1)

Zare, A., Mirrezapour, S.Z., Hallaji, M., Shoeibi, A., Jafari, M., Ghassemi, N., Alizadehsani, R., Mosavi, A. Robust adaptive synchronization of a class of uncertain chaotic systems with unknown time-delay (2020) Applied Sciences (Switzerland), 10 (24), art. no. 8875, pp. 1-14.

Baghban, A., Mosavi, A. Insight into the antiviral activity of synthesized schizonepetin derivatives: A theoretical investigation (2020) Scientific Reports, 10 (1), art. no. 8599 .

Pinter, G., Mosavi, A., Felde, I. Artificial intelligence for modeling real estate price using call detail records and hybrid machine learning approach (2020) Entropy, 22 (12), art. no. 1421, pp. 1-14.

Aram, F., Solgi, E., Garcia, E.H., Mosavi, A. Urban heat resilience at the time of global warming: evaluating the impact of the urban parks on outdoor thermal comfort (2020) Environmental Sciences Europe, 32 (1), art. no. 117, . 
Choubin, B., Borji, M., Hosseini, F.S., Mosavi, A., Dineva, A.A. Mass wasting susceptibility assessment of snow avalanches using machine learning models (2020) Scientific Reports, 10 (1), art. no. 18363, .

Gorji, N.E., Mosavi, A., Rouhi, A., Darvishzadeh, P., Ghadiri, M. Modeling Film Conductivity for Ion Migration Analysis in Perovskite Solar Cells (2020) Journal of Electronic Materials, 49 (12), pp. 7018-7023.

Mosavi, A., Soleimani, A., Karimi, A., Akbari, M., Karimipour, A., Karimipour, A. Investigating the effect of process parameters on the mechanical properties and temperature distribution in fiber laser welding of AISI304 and AISI 420 sheet using response surface methodology (2020) Infrared Physics and Technology, 111, art. no. 103478, .

Mosavi, A., Hekmatifar, M., Alizadeh, A., Toghraie, D., Sabetvand, R., Karimipour, A. The molecular dynamics simulation of thermal manner of Ar/Cu nanofluid flow: The effects of spherical barriers size (2020) Journal of Molecular Liquids, 319, art. no. 114183, .

Choubin, B., Hosseini, F.S., Fried, Z., Mosavi, A. Application of Bayesian Regularized Neural Networks for Groundwater Level Modeling (2020) CANDO-EPE 2020 - Proceedings, IEEE 3rd International Conference and Workshop in obuda on Electrical and Power Engineering, art. no. 9337753, pp. 209-212. https: / / www. scopus. com/inward/record.uri?eid=2-s2.085101103642 \&doi=10.1109\%2 fCANDOEPE51100.2020.9337753\&partner ID=40\&md5=fbfe39b898249e42aa7730e7da 35 f0f 9

Sedaghat, A., Band, S., Mosavi, A., Nadai, L. Predicting Covid-19 (Coronavirus Disease) Outbreak Dynamics Using SIR-based Models: Comparative Analysis of SIRD and Weibull-SIRD (2020) CANDO-EPE 2020 - Proceedings, IEEE 3rd International Conference and Workshop in Obuda on Electrical and Power Engineering, art. no. 9337791, pp. 283-288. https://www.scopus.com/inward/record.uri?eid=2-s2.085101103345 \&doi=10.11092 fCANDO-

EPE51100.2020.9337791\&partner ID=40\&md5=d9cd2a 04 e 73c8981db2f742055541460

Ardabili, S., Mosavi, A., Band, S.S., Varkonyi-Koczy, A.R. Coronavirus Disease (COVID-19) Global Prediction Using Hybrid Artificial Intelligence Method of ANN Trained with Grey Wolf Optimizer (2020) CANDO-EPE 2020 - Proceedings, IEEE 3rd International Conference and Workshop in Obuda on Electrical and Power Engineering, art. no. 9337757, pp. 251-254. https: / / www . scopus. com/inward/record.uri ?eid=2-s2.085101077412 \&doi=10.1109\%2 fCANDO-

EPE51100.2020.9337757 \&partner I $D=40$ \&md5=a 96250 f 82 e 4 e 76 ae 810 ba 4813 b 398 d 11

Sedaghat, A., Band, S., Mosavi, A., Nadai, L. Covid-19 (Coronavirus Disease) Outbreak Prediction Using a Susceptible-Exposed-Symptomatic Infected-RecoveredSuper Spreaders-Asymptomatic Infected-Deceased-Critical (SEIR-PADC) Dynamic Model (2020) CANDO-EPE 2020 - Proceedings, IEEE 3rd International Conference and Workshop in Obuda on Electrical and Power Engineering, art. no. 9337775, pp. 275-282.

https: / / www. scopus. com/inward/record.uri?eid=2-s2.0$85101077207 \&$ doi=10.1109\%2 fCANDO-

EPE51100.2020.9337775\&partnerID=40 \&md5=19cb4a2270523d0efe34 63 e8b09bbb8 9

Sedaghat, A., Band, S., Mosavi, A., Nadai, L. Predicting Trends of Coronavirus Disease (COVID-19) Using SIRD and Gaussian-SIRD Models (2020) CANDO-EPE 2020 - 
Proceedings, IEEE 3rd International Conference and Workshop in Obuda on Electrical and Power Engineering, art. no. 9337783, pp. 267-274.

https: / / www. scopus. com/inward/record.uri?eid=2-s2.0-

$85101068324 \&$ doi $=10.1109 \% 2$ fCANDO-

EPE51100.2020.9337783\&partner ID=40\&md5=3dffa 8563687 a 9 a 4 3cd21b1a 5482 e 901

Salimi, N., Rafe, V., Tabrizchi, H., Mosavi, A. Fuzzy Genetic Algorithm Approach for Verification of Reachability and Detection of Deadlock in Graph Transformation Systems (2020) CANDO-EPE 2020 - Proceedings, IEEE 3rd International Conference and Workshop in Obuda on Electrical and Power Engineering, art. no. 9337781, pp. 241-250. https: / / www. scopus. com/inward/record.uri?eid=2-s2.0$85101056192 \&$ do $i=10.1109 \% 2$ fCANDO-

EPE51100.2020.9337781\&partnerID=40\&md5=3869b60177e 7e 9efa295d206c1894d51

Tabrizchi, H., Mosavi, A., Szabo-Gali, A., Felde, I., Nadai, L. Rapid Covid-19 Diagnosis Using Deep Learning of the Computerized Tomography Scans (2020)

CANDO-EPE 2020 - Proceedings, IEEE 3rd International Conference and Workshop in Obuda on Electrical and Power Engineering, art. no. 9337794, pp. 173-178. https: //www. scopus. com/inward/record.uri?eid=2-s2.0$85101059817 \&$ doi=10.1109\%2 fCANDO-

EPE51100 . 2020.9337794 \&partner I $D=40$ \&md5=2c12 cb7 7b5bbaa 60 b 4942 dad1d855f36

Sedaghat, A., Oloomi, S.A.A., Malayer, M.A., Band, S., Mosavi, A., Nadai, L. Modeling and Sensitivity Analysis of Coronavirus Disease (COVID-19) Outbreak Prediction (2020) CANDO-EPE 2020 - Proceedings, IEEE 3rd International Conference and Workshop in Obuda on Electrical and Power Engineering, art. no. 9337772, pp. 261-266.

https://www. scopus. com/inward/record.uri?eid=2-s2.0$85101070027 \&$ doi $=10.1109 \% 2$ fCANDO-

EPE51100.2020.9337772\&partner ID=40\&md5=ac3d3b17451 c5ff2 fedlde 4 bd96e2dcc

Sedaghat, A., Oloomi, S.A.A., Malayer, M.A., Band, S., Rezaei, N., Mosavi, A., Nadai, L. Coronavirus (COVID-19) Outbreak Prediction Using Epidemiological Models of Richards Gompertz Logistic Ratkowsky and SIRD (2020) CANDO-EPE 2020 Proceedings, IEEE 3rd International Conference and Workshop in Obuda on Electrical and Power Engineering, art. no. 9337799, pp. 289-298. https: / / www. scopus. com/inward/record.uri ?eid=2-s2.0$85101077917 \&$ doi=10.1109\%2 fCANDO-

EPE51100.2020.9337799\&partner ID=40\&md5=6535741f1465e5e2b8694a 7812 e 93 fdd

D'Orazio, A., Karimipour, A., Mosavi, A. Develop lattice Boltzmann method and its related boundary conditions models for the benchmark oscillating walls by modifying hydrodynamic and thermal distribution functions (2020) European Physical Journal Plus, 135 (11), art. no. 915, . https: //www. scopus. com/inward/record.uri ?eid=2-s2.0$85096175643 \& d o i=10.1140 \div 2$ fepjp $\% 2$ fs $13360-020-00925-$ 4 \&partnerI $=40$ \&md5=9e 4 d29 c 9 b $79 b 6325$ dfeb 2 f c 5 fe 9 a 4 ef

Ecer, F., Ardabili, S., Band, S.S., Mosavi, A. Training multilayer perceptron with genetic algorithms and particle swarm optimization for modeling stock price index prediction (2020) Entropy, 22 (11), art. no. 1239, pp. 1-20. https: / / www. scopus. com/inward/record.uri?eid=2-s2.085094876535 \&do $i=10.3390 \% 2 \mathrm{fe} 2111239 \&$ partner $\mathrm{I} D=40$ \&md5=589e545f $66 \mathrm{da} 5 \mathrm{~b} 1 \mathrm{~d} 7 \mathrm{bed} 01698 \mathrm{e}$ $65 f 321$

Zandi, P., Rahmani, M., Khanian, M., Mosavi, A. Agricultural risk management using fuzzy topsis analytical hierarchy process (Ahp) and failure mode and 
effects analysis (fmea) (2020) Agriculture (Switzerland), 10 (11), art. no. 504, pp. $1-28$.

https://www.scopus.com/inward/record.uri?eid=2-s2.0-

$85094572320 \& d o i=10.3390 \% 2$ fagriculture 10110504 \&partner $\mathrm{D}=40$ \&md5=59e 6 e $49 \mathrm{c} 9588333 \mathrm{c}$ $20123 \mathrm{~b} 70 \mathrm{c} 40 \mathrm{c} 1384$

Claywell, R., Nadai, L., Felde, I., Ardabili, S., Mosavi, A. Adaptive neurofuzzy inference system and a multilayer perceptron model trained with grey wolf optimizer for predicting solar diffuse fraction (2020) Entropy, 22 (11), art. no. 1192, pp. 1-14.

https: //www.scopus.com/inward/record.uri?eid=2-s2.0-

85093964080 \&do $i=10.3390 \% 2 \mathrm{fe} 22111192$ \&partner $I D=40 \& \mathrm{md} 5=2656 \mathrm{bb} 70216 \mathrm{bfbc} 660 \mathrm{fe} 3 \mathrm{cc} 8 \mathrm{~b} 7$ cecblc

Karballaeezadeh, N., Danial, M.S., Moazemi, D., Band, S.S., Mosavi, A., Reuter, U. Smart structural health monitoring of flexible pavements using machine learning methods (2020) Coatings, 10 (11), art. no. 1100, pp. 1-18. https://www. scopus.com/inward/record.uri?eid=2-s2.085096299941 \&do $i=10.3390 \% 2$ fcoatings 10111100 \&partner $I D=40$ \& md $5=4199 \mathrm{aff} 2 \mathrm{aeb} 862 \mathrm{c} 3 \mathrm{c} 0 \mathrm{e}$ 2 f69446eb66f 8

Band, S.S., Al-Shourbaji, I., Karami, H., Karimi, S., Esfandiari, J., Mosavi, A. Combination of group method of data handling (GMDH) and computational fluid dynamics (CFD) for prediction of velocity in channel intake (2020) Applied Sciences (Switzerland), 10 (21), art. no. 7521, pp. 1-15. https: //www. scopus.com/inward/record.uri?eid=2-s2.085094641589 \&do $i=10.3390 \% 2$ fapp 10217521 \&partner $\mathrm{D}=40$ \&md5 =854 4 c5794 dae 58635 ec 6 de 76 bdoef165

Bonakdari, H., Gholami, A., Mosavi, A., Kazemian-Kale-Kale, A., Ebtehaj, I., Azimi, A.H. A novel comprehensive evaluation method for estimating the bank profile shape and dimensions of stable channels using the maximum entropy principle (2020) Entropy, 22 (11), art. no. 1218, pp. 1-23. https://www. scopus.com/inward/record.uri?eid=2-s2.085094557206 \&do $i=10.3390 \% 2$ fe22111218 \&partner $I D=40$ \&md5=e 969 fd8 75 caf 539 d3 62 fd82024 $5 \mathrm{a} 9 \mathrm{c} 4 \mathrm{~b}$

Band, S.S., Janizadeh, S., Pal, S.C., Saha, A., Chakrabortty, R., Melesse, A.M., Mosavi, A. Flash flood susceptibility modeling using new approaches of hybrid and ensemble tree-based machine learning algorithms

(2020) Remote Sensing, 12 (21), art. no. 3568, pp. 1-23. https: //www. scopus.com/inward/record.uri?eid=2-s2.0-

85094912774 \&do $i=10.3390 \% 2 \mathrm{frs} 12213568$ \&partner $I D=40$ \&ma $5=4$ da 19 e0 $0336 \mathrm{~b} 1$ af $104 \mathrm{~b} 7 \mathrm{fbf} 588$ a $637 \mathrm{~b} 51$

Mostafaeipour, A., Fakhrzad, M.B., Gharaat, S., Jahangiri, M., Dhanraj, J.A., Band, S.S., Issakhov, A., Mosavi, A. Machine learning for prediction of energy in wheat production (2020) Agriculture (Switzerland), 10 (11), art. no. 517, pp. $1-18$.

https://www.scopus.com/inward/record.uri?eid=2-s2.0-

85094821186 \&do $i=10.3390 \% 2$ fagriculture 10110517 \&partner $I D=40$ \&md5=32341 caae $02 \mathrm{c} 1447$ $241 \mathrm{a} 28 \mathrm{fa} 5 \mathrm{e} 9 \mathrm{bd} 25 \mathrm{e}$

Samadianfard, S., Hashemi, S., Kargar, K., Izadyar, M., Mostafaeipour, A., Mosavi, A., Nabipour, N., Shamshirband, S. Wind speed prediction using a hybrid model of the multi-layer perceptron and whale optimization algorithm

(2020) Energy Reports, 6, pp. 1147-1159. 
https: / / www. scopus. com/inward/record.uri?eid=2-s2.0$85084258687 \& d o i=10.1016 \% 2 \mathrm{fj}$. egyr. $2020.05 .001 \&$ partner $\mathrm{D}=40$ \&md5=2706 ce $6 \mathrm{c} 14 \mathrm{ff} 08095$ $23 f 189 \mathrm{bb} 2832 \mathrm{a} 0 \mathrm{a}$

Salcedo-Sanz, S., Ghamisi, P., Piles, M., Werner, M., Cuadra, L., MorenoMartínez, A., Izquierdo-Verdiguier, E., Muñoz-Marí, J., Mosavi, A., CampsValls, G. Machine learning information fusion in Earth observation: A comprehensive review of methods, applications and data sources (2020) Information Fusion, 63, pp. 256-272. https: / / www . scopus. com/inward/record.uri?eid=2-s2.0$85088030361 \&$ doi=10.1016\%2fj.inffus. $2020.07 .004 \&$ partner $\mathrm{D}=40 \& \mathrm{md} 5=4956874 \mathrm{feeb} 475 \mathrm{a}$ $8 \mathrm{~b} 6 \mathrm{f} 0512 \mathrm{~b} 120700 \mathrm{a} 9$

Akhoundi, B., Nabipour, M., Hajami, F., Band, S.S., Mosavi, A. Calculating filament feed in the fused deposition modeling process to correctly print continuous fiber composites in curved paths (2020) Materials, 13 (20), art. no. 4480 , pp. 1-11.

https://www. scopus. com/inward/record.uri?eid=2-s2 . $0-$

$85093944527 \&$ doi=10.3390\%2 fma13204480 \&partner ID=4 0 \&md5=15f966149d3462fb239066ac 7 d7 c39a 8

Band, S.S., Janizadeh, S., Pal, S.C., Chowdhuri, I., Siabi, Z., Norouzi, A., Melesse, A.M., Shokri, M., Mosavi, A. Comparative analysis of artificial intelligence models for accurate estimation of groundwater nitrate concentration (2020) Sensors (Switzerland), 20 (20), art. no. 5763, pp. 1-23. https: / / www . scopus. com/inward/record.uri ?eid=2-s2 . 085092665947 \&doi=10.3390\%2 fs 20205763 \&partner $\mathrm{D}=40$ \&md5=2bb7 $1 \mathrm{a} 5 \mathrm{~d} 47332 \mathrm{a} 2 \mathrm{~b} 3879 \mathrm{~d} 5305 \mathrm{~b}$ c7e4a 2

Karimimoshaver, M., Ahmadi, M.A., Aram, F., Mosavi, A. Urban views and their impacts on citizens: A grounded theory study of Sanandaj city (2020) Heliyon, 6 (10), art. no. e05157, .

https: / / www. scopus. com/inward/record.uri?eid=2-s2.0-

85092192616 \&doi=10.1016\%2fj.heliyon. 2020 .e 05157 \&partner $\mathrm{D}=40$ \&md5=a $9 \mathrm{e} 0 \mathrm{f} 88 \mathrm{ff} 3 \mathrm{f} 859$ 367277 de 6 f04d3c237

Nabiollahi, K., Heshmat, E., Mosavi, A., Kerry, R., Zeraatpisheh, M., Taghizadeh-Mehrjardi, R. Assessing the influence of soil quality on rainfedwheat yield (2020) Agriculture (Switzerland), 10 (10), art. no. 469, pp. $1-18$.

https: / / www . scopus. com/inward/record.uri ?eid=2-s2 . $0-$

85092481372 \&do $i=10.3390 \% 2$ fagriculture 10100469 \&partner $\mathrm{D}=40$ \&md5=877de 5 e2 5 dd $6 \mathrm{~b} 9 \mathrm{e} 8$ d 4 eb 7 e $028 \mathrm{dbb} 42 \mathrm{dd}$

Ardabili, S., Beszedes, B., Nadai, L., Szell, K., Mosavi, A., Imre, F. Comparative Analysis of Single and Hybrid Neuro-Fuzzy-Based Models for an Industrial Heating Ventilation and Air Conditioning Control system (2020) Proceedings - 2020 RIVF International Conference on Computing and Communication Technologies, RIVF 2020, art. no. 9140753, . https: / / www. scopus. com/inward/record.uri?eid=2-s2.0$85090270726 \& \mathrm{do}=10.1109 \% 2 \mathrm{fRIVF} 48685.2020 .9140753 \&$ partner $\mathrm{D}=40 \& \mathrm{md} 5=\mathrm{a} 93 \mathrm{fd} 7464 \mathrm{a} 1 \mathrm{df}$ bcf7001f98b499174b9

Nosratabadi, S., Szell, K., Beszedes, B., Imre, F., Ardabili, S., Mosavi, A. Comparative Analysis of ANN-ICA and ANN-GWO for Crop Yield Prediction (2020) Proceedings - 2020 RIVF International Conference on Computing and Communication Technologies, RIVF 2020, art. no. 9140786, . 
https: / / www. scopus. com/inward/record.uri?eid=2-s2.0$85090269380 \&$ doi=10 . $1109 \% 2$ fRIVF $48685.2020 .9140786 \&$ partner $\mathrm{D}=40 \& \mathrm{md5}=03684 \mathrm{da} 7965 \mathrm{~b} 8$ dac 9666289 ec 50 ecedc

Nadai, L., Imre, F., Ardabili, S., Gundoshmian, T.M., Gergo, P., Mosavi, A. Performance Analysis of Combine Harvester using Hybrid Model of Artificial Neural Networks Particle Swarm Optimization (2020) Proceedings - 2020 RIVF International Conference on Computing and Communication Technologies, RIVF 2020, art. no. 9140748, . https: / / www. scopus. com/inward/record.uri?eid=2-s2.085086125288 \&doi=10.1109\%2 fRIVF 48685.2020 .9140748 \&partner ID=40 \&md5=8069e4a 50723c f1b2ba5c3a170eb700f

Melesse, A.M., Khosravi, K., Tiefenbacher, J.P., Heddam, S., Kim, S., Mosavi, A., Pham, B.T. River water salinity prediction using hybrid machine learning models (2020) Water (Switzerland), 12 (10), art. no. 2951, pp. 1-21. https: / / www. scopus. com/inward/record.uri?eid=2-s2.085095973848 \&do $i=10.3390 \% 2 \mathrm{fw} 12102951$ \&partner $\mathrm{ID}=40$ \&md5=8ec $652 \mathrm{~d} 636 \mathrm{~b} 50 \mathrm{~d} 787 \mathrm{a} 3 \mathrm{e} 81873 \mathrm{f}$ $5 \mathrm{~d} 0 \mathrm{f} 30$

Mosavi, A., Hosseini, F.S., Choubin, B., Abdolshahnejad, M., Gharechaee, H., Lahijanzadeh, A., Dineva, A.A. Susceptibility prediction of groundwater hardness using ensemble machine learning models (2020) Water (Switzerland), 12 (10), art. no. 2770, . https: / / www. scopus. com/inward/record.uri ?eid=2-s2.0$85092719343 \& \mathrm{do} i=10.3390 \% 2 \mathrm{fw} 12102770$ \&partner $\mathrm{D}=40$ \&md5=cfa 1 edef $1 \mathrm{fc} 591 \mathrm{c} 2 \mathrm{e} 61 \mathrm{db} 838 \mathrm{~d} 1$ 66 bdeb

Mosavi, A., Faghan, Y., Ghamisi, P., Duan, P., Ardabili, S.F., Salwana, E., Band, S.S. Comprehensive review of deep reinforcement learning methods and applications in economics (2020) Mathematics, 8 (10), art. no. 1640, . https: / / www . scopus. com/inward/record.uri?eid=2-s2 . 0$85092487643 \&$ doi=10 $.3390 \% 2$ fMATH 8101640 \&partnerID=4 0 \&md5=a $73687 \mathrm{c} 4 \mathrm{fec} 5 \mathrm{e} 7329536 \mathrm{~d} 4 \mathrm{CC}$ bc5b6acf

Band, S.S., Janizadeh, S., Pal, S.C., Saha, A., Chakrabortty, R., Shokri, M., Mosavi, A. Novel ensemble approach of deep learning neural network (Dlnn) model and particle swarm optimization (pso) algorithm for prediction of gully erosion susceptibility (2020) Sensors (Switzerland), 20 (19), art. no. 5609, pp. 1-28. https: / / www. scopus. com/inward/record.uri?eid=2-s2.0-

$85091873905 \&$ do $i=10.3390 \% 2$ fs2 $0195609 \&$ partner $I D=40 \& \mathrm{md} 5=797948866 e 3 f 4982 \mathrm{c} 872994002$ $898 \mathrm{a} 66$

Mojrian, S., Pinter, G., Joloudari, J.H., Felde, I., Szabo-Gali, A., Nadai, L., Mosavi, A. Hybrid Machine Learning Model of Extreme Learning Machine Radial basis function for Breast Cancer Detection and Diagnosis; A Multilayer Fuzzy Expert System (2020) Proceedings - 2020 RIVF International Conference on Computing and Communication Technologies, RIVF 2020, art. no. 9140744, . https: / / www. scopus. com/inward/record.uri?eid=2-s2.0$85090272819 \&$ doi=10.1109\%2fRIVF $48685.2020 .9140744 \&$ partner ID $=40 \& \mathrm{md} 5=\mathrm{e} 918 \mathrm{bfc} 4 \mathrm{cbf} 3 \mathrm{~d}$ e3319786903501cldie

Band, S.S., Janizadeh, S., Saha, S., Mukherjee, K., Bozchaloei, S.K., Cerdà, A., Shokri, M., Mosavi, A. Evaluating the efficiency of different regression, decision tree, and bayesian machine learning algorithms in spatial piping erosion susceptibility using alos/palsar data (2020) Land, 9 (10), art. no. 346 , pp. 1-22. 
https: / / www. scopus. com/inward/record.uri?eid=2-s2.0$85093671485 \& d o i=10.3390 \% 2 \mathrm{fland} 9100346$ \&partner $\mathrm{D}=40$ \&md5=11ec2baa $4630727 \mathrm{~b} 05531 \mathrm{e} 12$ $5925 \mathrm{~d} 0 \mathrm{aa}$

Mohamadi, S., Sammen, S.S., Panahi, F., Ehteram, M., Kisi, O., Mosavi, A., Ahmed, A.N., El-Shafie, A., Al-Ansari, N. Zoning map for drought prediction using integrated machine learning models with a nomadic people optimization algorithm (2020) Natural Hazards, 104 (1), pp. 537-579. https: / / www . scopus. com/inward/record.uri?eid=2-s2 . 0$85089579917 \& d o i=10.1007 \% 2$ fs11069-020-04180-

9 \&partner $I D=40$ \&md $5=\mathrm{b} 48189$ a 8 baa 7938 c 3 ded 4 a ac 3 eea 00 f 0

Nosratabadi, S., Mosavi, A., Duan, P., Ghamisi, P., Filip, F., Band, S.S., Reuter, U., Gama, J., Gandomi, A.H. Data science in economics: Comprehensive review of advanced machine learning and deep learning methods (2020)

Mathematics, 8 (10), art. no. 1799, pp. 1-25.

https: / / www. scopus. com/inward/record.uri?eid=2-s2.0-

85086140360 \&do $i=10.3390 \% 2$ math $8101799 \&$ partner $\mathrm{D}=40$ \&md5=1 c 8 af 62 eb $552 \mathrm{ab} 6906 \mathrm{f} 2 \mathrm{aef} 2$ $5 e c 16 f 1 e$

Abbasi, S., Jannaty, M.H., Faraj, R.H., Shahbazpanahi, S., Mosavi, A. The effect of incorporating silica stone waste on the mechanical properties of sustainable concretes (2020) Materials, 13 (17), art. no. 3832, . https: / / www . scopus. com/inward/record.uri?eid=2-s2.0-

85090933764 \&doi=10.3390\%2 fma 13173832 \&partner $\mathrm{ID}=40$ \&md5=54 cb $2 \mathrm{ab} 179 \mathrm{c} 04 \mathrm{~b} 970 \mathrm{a} 7 \mathrm{fcaef \textrm {c }}$ $61 \mathrm{ad} 3 \mathrm{c} 0$

Mosavi, A., Qasem, S.N., Shokri, M., Shahab, S., Mohammadzadeh, A. Fractionalorder fuzzy control approach for photovoltaic/battery systems under unknown dynamics, variable irradiation and temperature (2020) Electronics

(Switzerland), 9 (9), art. no. 1455, pp. 1-19. https: / / www. scopus. com/inward/record.uri?eid=2-s2.085090291670 \&doi=10.3390\%2 felectronics 9091455 \&partner $\mathrm{D}=40$ \&md5=b60bc 9 e $79 \mathrm{aa} 347 \mathrm{f} 77$ e5618067361389b

Mosavi, A., Shokri, M., Mansor, Z., Qasem, S.N., Band, S.S., Mohammadzadeh, A. Machine learning for modeling the singular multi-pantograph equations (2020) Entropy, 22 (9), art. no. 1041,.

https: / / www. scopus. com/inward/record.uri?eid=2-s2.085092542968 \&doi=10 . 3390\%2fE22091041\&partner ID=40\&md5=93605478fdbe 5477983 f c $5 \mathrm{c} 7 \mathrm{a} 5$ $9 \mathrm{~d} 6 \mathrm{~b} 5 \mathrm{~b}$

Mosavi, A., Mehdizadeh, H., Abbasian-Naghneh, S., Kalbasi, R., Karimipour, A., Cheraghian, G. Incorporation of horizontal fins into a PCM-Based heat sink to enhance the safe operation time: Applicable in electronic device cooling (2020) Applied Sciences (Switzerland), 10 (18), art. no. 6308, . https: / / www. scopus. com/inward/record.uri?eid=2-s2.0-

85091846319 \&doi=10.3390\%2 fAPP1 0186308 \&partner $\mathrm{D}=40$ \&md5=1a989d0bdc56b92a 08 e 1 b $3 b 3$ a 564 a $79 d$

Yousefi, Y., Karballaeezadeh, N., Moazami, D., Zahed, A.S., Danial Mohammadzadeh, S., Mosavi, A. Improving aviation safety through modeling accident risk assessment of runway (2020) International Journal of Environmental Research and Public Health, 17 (17), art. no. 6085, pp. 1-36. https: / / www. scopus. com/inward/record.uri?eid=2-s2.085089794755 \&doi=10.3390\%2fijerph17176085\&partner $\mathrm{D}=40$ \&md5=fe1d0a2517602 fd3e $79 \mathrm{cf}$ 8 f 5 f 20 fc 0 e 6 
Dodangeh, E., Singh, V.P., Pham, B.T., Yin, J., Yang, G., Mosavi, A. Flood Frequency Analysis of Interconnected Rivers by Copulas (2020) Water Resources Management, 34 (11), pp. 3533-3549.

https://www.scopus.com/inward/record.uri?eid=2-s2.0-

$85088844557 \& d o i=10.1007 \% 2$ fs11269-020-02634-

0 \&partner $I D=40$ \&md $5=361405 \mathrm{c} 9 \mathrm{a} 8 \mathrm{~d} 92 \mathrm{~d} 1 \mathrm{~d} 1 \mathrm{fddde} 60 \mathrm{ff} 0 \mathrm{e} 5 \mathrm{~b} 1 \mathrm{c}$

Shateri, M., Sobhanigavgani, Z., Alinasab, A., Varamesh, A., Hemmati-

Sarapardeh, A., Mosavi, A., Shamshirband, S.S. Comparative analysis of machine

learning models for nanofluids viscosity assessment (2020) Nanomaterials, 10

(9), art. no. 1767, pp. 1-22.

https://www.scopus.com/inward/record.uri?eid=2-s2.0-

$85090353635 \&$ do $i=10$. $3390 \% 2$ fnano 10091767 \&partner I $D=40$ \&md5=bbd2a 812 ab5 299 ea fee 1 e 04

93 be 56146

Mosavi, A., Gorji, N.E. Brief review on thin films, perovskite solar cells and nanostructure's applications (2020) Modern Physics Letters B, 34 (24), art. no. 2030003,.

https://www. scopus.com/inward/record.uri?eid=2-s2.0-

$85091433381 \&$ do $i=10.1142 \% 2$ fS $0217984920300033 \&$ partner I $=40$ \&md5=365958cd2ec $737 \mathrm{bd} 04$ 878 aa424492047

Ameli, A., Maher, J., Mosavi, A., Nabipour, N., Babagoli, R., Norouzi, N. Performance evaluation of binders and Stone Matrix Asphalt (SMA) mixtures modified by Ground Tire Rubber (GTR), waste Polyethylene Terephthalate (PET) and Anti Stripping Agents (ASAs) (2020) Construction and Building Materials, 251, art. no. 118932, .

https: //www.scopus.com/inward/record.uri?eid=2-s2.0-

$85082778334 \&$ do $i=10.1016 \% 2 \mathrm{fj}$. conbuildmat. $2020.118932 \&$ partner $I D=40$ \&md5=249e77aa51 90 eb $9 d 6 b 1 b d a 3 f 4269 f 21 a$

Nasiri, A.S.A., Rahmani, O., Kordani, A.A., Karballaeezadeh, N., Mosavi, A. Evaluation of safety in horizontal curves of roads using a multi-body dynamic simulation process (2020) International Journal of Environmental Research and Public Health, 17 (16), art. no. 5975, pp. 1-20.

https://www.scopus.com/inward/record.uri?eid=2-s2.0-

$85089664096 \&$ do $i=10$. 3390\%2fijerph17165975 \&partner I $=40$ \&md5=d19afc99c1241b1a5a 7 a 7 $2 \mathrm{a} 64061 \mathrm{bfbe}$

Habibi, K., Hoseini, S.M., Dehshti, M., Khanian, M., Mosavi, A. The impact of natural elements on environmental comfort in the iranian-islamic historical city of Isfahan (2020) International Journal of Environmental Research and Public Health, 17 (16), art. no. 5776, pp. 1-18.

https: //www. scopus.com/inward/record.uri?eid=2-s2.0-

85089344252 \&do $i=10.3390 \% 2$ fijerph 17165776 \&partner $I D=40$ \&md5=59f 8 b3bfca $8 \mathrm{c} 2405$ fac 94 c $63 \mathrm{~d} 372 \mathrm{c} 986$

Shamshirband, S., Mosavi, A., Rabczuk, T. Particle swarm optimization model to predict scour depth around a bridge pier (2020) Frontiers of Structural and Civil Engineering, 14 (4), pp. 855-866.

https://www. scopus.com/inward/record.uri?eid=2-s2.0-

$85086852956 \&$ do $i=10.1007 \% 2$ fs11709-020-0619-

2 \&partner $I D=40$ \&md5=3ebe $745 \mathrm{~b} 736 \mathrm{e} 9 \mathrm{e} 18 \mathrm{e} 0 \mathrm{~b}$ f $1 \mathrm{~b} 6 \mathrm{ee} 9 \mathrm{c} 08 \mathrm{~b} 2 \mathrm{~d}$

Sheikh Khozani, Z., Khosravi, K., Torabi, M., Mosavi, A., Rezaei, B., Rabczuk, T. Shear stress distribution prediction in symmetric compound channels using data mining and machine learning models (2020) Frontiers of Structural and Civil Engineering, 14 (5), pp. 1097-1109. 
https://www.scopus.com/inward/record.uri?eid=2-s2.0$85098454598 \& d o i=10.1007 \% 2$ fs11709-020-0634-

3 \&partnerI $D=40$ \&md5=d03dda $6 a 4063958 \mathrm{~d} 104 \mathrm{f} 1 \mathrm{cda} 1303 \mathrm{~b} 29 \mathrm{~d}$

Nabipour, M., Nayyeri, P., Jabani, H., Mosavi, A., Salwana, E., Shahab, S. Deep learning for stock market prediction (2020) Entropy, 22 (8), art. no. 840, https: / / www. scopus. com/inward/record.uri?eid=2-s2.0$85089841373 \& d o i=10.3390 \% 2$ fE2 2080840 \&partner $\mathrm{D}=40$ \&md5=1d $8 \mathrm{db} f 047 \mathrm{ac} 9 \mathrm{~b} 21$ eeddc $10 \mathrm{f} 1 \mathrm{e} 5$ a 4 c556

Lei, X., Chen, W., Avand, M., Janizadeh, S., Kariminejad, N., Shahabi, H., Costache, R., Shahabi, H., Shirzadi, A., Mosavi, A.

GIS-based machine learning algorithms for gully erosion susceptibility mapping in a semi-arid region of Iran (2020) Remote Sensing, 12 (15), art. no. 2478, .

https: / / www. scopus. com/inward/record.uri?eid=2-s2.0-

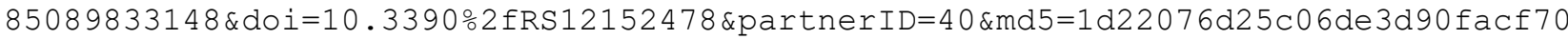
ace $81 \mathrm{fa}$

Sahragard, A., Falaghi, H., Farhadi, M., Mosavi, A., Estebsari, A. Generation expansion planning in the presence of wind power plants using a genetic algorithm model

(2020) Electronics (Switzerland), 9 (7), art. no. 1143, pp. 1-23. https: / / www. scopus. com/inward/record.uri?eid=2-s2.0-

85087952095 \&doi=10 . 3390\%2 felectronics 9071143 \&partner $\mathrm{D}=40$ \&md5=3a $89 \mathrm{c} 3824$ ea 30 e 158 ce 65 ef $3 \mathrm{dcb} 0 \mathrm{a} 8 \mathrm{~b} 5$

Mosavi, A., Sajedi-Hosseini, F., Choubin, B., Taromideh, F., Rahi, G., Dineva, A.A.

Susceptibility mapping of soil water erosion using machine learning models (2020) Water (Switzerland), 12 (7), art. no. 1995,. https: / / www. scopus. com/inward/record.uri?eid=2-s2.0$85087969016 \& d o i=10.3390 \% 2 f w 12071995$ \&partner $I D=40 \& m d 5=79 \mathrm{c} 88 \mathrm{~b} 104$ f 5 f $9 \mathrm{a} 575 \mathrm{dea} 896 \mathrm{~d} 17$ $660 \mathrm{afl}$

Emadi, M., Taghizadeh-Mehrjardi, R., Cherati, A., Danesh, M., Mosavi, A., Scholten, T.

Predicting and mapping of soil organic carbon using machine learning algorithms in Northern Iran

(2020) Remote Sensing, 12 (14), art. no. 2234,. https: / / www. scopus. com/inward/record.uri?eid=2-s2.0-

85087956866 \&doi=10.3390\%2 frs 12142234 \&partner ID=4 0 \&md5=a $79 \mathrm{c} 90 \mathrm{~b} 4 \mathrm{e} 0$ fee 8 fee $03 \mathrm{e} 86472$ 590 fec 6

Chahardowli, M., Sajadzadeh, H., Aram, F., Mosavi, A. Survey of sustainable regeneration of historic and cultural cores of cities (2020) Energies, 13 (11), art. no. 2708,. https: / / www. scopus. com/inward/record.uri?eid=2-s2.085085938784 \&do $i=10.3390 \% 2$ fen 13112708 \&partner $I D=40$ \&md5=9960400b3ea $1112 \mathrm{da} 2 \mathrm{ed} 7 \mathrm{~b} 79 \mathrm{a}$ $5 \mathrm{dd} 3 \mathrm{~d} 04$

Zhang, D., Ashraf, M.A., Liu, Z., Peng, W.-X., Golkar, M.J., Mosavi, A. Dynamic modeling and adaptive controlling in GPS-intelligent buoy (GIB) systems based on neural-fuzzy networks

(2020) Ad Hoc Networks, 103, art. no. 102149,. 
https: / / www. scopus. com/inward/record.uri?eid=2-s2.0-

$85083510764 \& d o i=10.1016 \% 2 \mathrm{fj}$. adhoc $.2020 .102149 \&$ partner $\mathrm{D}=40 \& \mathrm{md} 5=0 \mathrm{~cd} 981 \mathrm{~d} 03198 \mathrm{c} 791$ $49 e 6$ f 6 d5 59 e 65 e 4 e

Ashrafian, A., Taheri Amiri, M.J., Masoumi, P., Asadi-Shiadeh, M., YaghoubiChenari, M., Mosavi, A., Nabipour, N.

Classification-based regression models for prediction of the mechanical

properties of roller-compacted concrete pavement

(2020) Applied Sciences (Switzerland), 10 (11), art. no. 3707, .

https: / / www. scopus. com/inward/record.uri?eid=2-s2.0-

$85086122940 \& \mathrm{doi}=10.3390 \% 2 \mathrm{fapp} 10113707 \&$ partnerI $=40$ \&md5=dc2 f $687 \mathrm{~d} 4951405121689 \mathrm{~b} 6 \mathrm{f}$

$77 \mathrm{f} 87 \mathrm{ba} 8$

Ahmad, Z., Zhong, H., Mosavi, A., Sadiq, M., Saleem, H., Khalid, A., Mahmood, S., Nabipour, N.

Machine learning modeling of aerobic biodegradation for azo dyes and hexavalent chromium

(2020) Mathematics, 8 (6), art. no. 913,.

https: / / www. scopus. com/inward/record.uri?eid=2-s2.0-

$85087462805 \&$ do $i=10.3390 \% 2$ fMATH $8060913 \&$ partnerID=40\&md5=62d742ece 77 f $3 \mathrm{~d} 2 \mathrm{a} 00 \mathrm{a} 0 \mathrm{c} 907$ 0 caf 6709

Seifi, A., Ehteram, M., Singh, V.P., Mosavi, A.

Modeling and uncertainty analysis of groundwater level using six evolutionary optimization algorithms hybridized with ANFIS, SVM, and ANN

(2020) Sustainability (Switzerland), 12 (10), art. no. 4023, .

https: / / www. scopus. com/inward/record.uri?eid=2-s2.0-

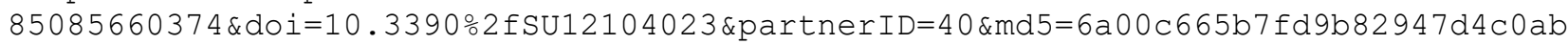
$6 \mathrm{~b} 5027 \mathrm{a}$

Khakian, R., Karimimoshaver, M., Aram, F., Benis, S.Z., Mosavi, A., VarkonyiKoczy, A.R.

Modeling nearly zero energy buildings for sustainable development in rural

areas

(2020) Energies, 13 (10), art. no. 2593, .

https: / / www. scopus. com/inward/record.uri ?eid=2-s2.0-

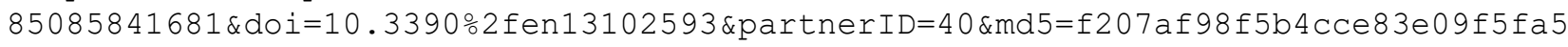
$8 \mathrm{c} 8 \mathrm{~d} 601$

Rezaei, A., Abdollahi, H., Derikvand, Z., Hemmati-Sarapardeh, A., Mosavi, A., Nabipour, N.

Insights into the effects of pore size distribution on the flowing behavior of carbonate rocks: Linking a nano-based enhanced oil recovery method to rock

typing

(2020) Nanomaterials, 10 (5), art. no. 972, .

https: / / www. scopus. com/inward/record.uri?eid=2-s2.0-

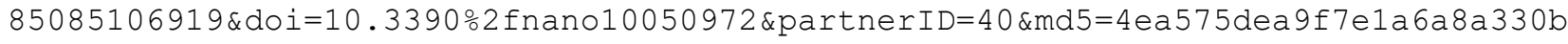
$15 \mathrm{cb} 0 \mathrm{~d} 78 \mathrm{~d}$

Mosavi, A., Bertalan, B., Imre, F., Nadai, L., Gorji, N.E. Electrical characterization of CIGS thin-film solar cells by two- and four-wire probe technique

(2020) Modern Physics Letters B, 34 (11), art. no. 2050102, .

https: / / www. scopus. com/inward/record.uri?eid=2-s2.0-

$85078980082 \&$ do $i=10.1142 \% 2$ fS $021798492050102 x \&$ partnerI $=40 \& \mathrm{md} 5=\mathrm{a} 98 \mathrm{e} 44643 \mathrm{bddd} 5666 \mathrm{a}$ $5 e 735797 d 7 e 782$

Jilte, R., Ahmadi, M.H., Kumar, R., Kalamkar, V., Mosavi, A. 
Cooling performance of a novel circulatory flow concentric multi-channel heat sink with nanofluids

(2020) Nanomaterials, 10 (4), art. no. 647, .

https://www. scopus.com/inward/record.uri?eid=2-s2.0-

85083305841 \&doi=10.3390\%2 fnano 10040647 \&partner I $=40$ \&md5=1c330b 37 fbe 6 f $948 \mathrm{bf} 6009 \mathrm{c}$

$7 \mathrm{~b} 6 \mathrm{a} 5 \mathrm{c} 282$

Bemani, A., Baghban, A., Shamshirband, S., Mosavi, A., Csiba, P., VarkonyiKoczy, A.R.

Applying ANN, ANFIS and LSSVM models for estimation of acid solvent solubility in supercritical $\mathrm{CO} 2$

(2020) Computers, Materials and Continua, 63 (3), pp. 1175-1204.

https: / /www. scopus.com/inward/record.uri?eid=2-s2.0-

$85091090813 \& \mathrm{do} i=10.32604 \% 2 \mathrm{fCMC} .2020 .07723$ \&partner $\mathrm{D}=40$ \&ma5=a $809164 \mathrm{bf} 3 \mathrm{ff} 6 \mathrm{e} 1 \mathrm{~b} 11 \mathrm{e} 3$ $3 \mathrm{~d} 09 \mathrm{f} 0 \mathrm{~d} 7077 \mathrm{~b}$

Sadeghzadeh, M., Maddah, H., Ahmadi, M.H., Khadang, A., Ghazvini, M., Mosavi, A., Nabipour, N.

Prediction of Thermo-Physical Properties of TiO2-Al203/Water Nanoparticles by Using Artificial Neural Network

(2020) Nanomaterials, 10 (4), art. no. 697, .

https: //www. scopus.com/inward/record.uri?eid=2-s2.0-

85083853257 \&do $i=10.3390 \% 2$ fnano 10040697 \&partner $I D=40$ \&md5=6d0 $7 \mathrm{~d} 9 \mathrm{fc} 56 \mathrm{ba} 907 \mathrm{f} 7 \mathrm{~d} 9 \mathrm{c} 688$ $1 c 2957444$

Fathi, S., Sajadzadeh, H., Sheshkal, F.M., Aram, F., Pinter, G., Felde, I., Mosavi, A.

The role of urban morphology design on enhancing physical activity and public health

(2020) International Journal of Environmental Research and Public Health, 17

(7), art. no. 2359, .

https://www.scopus.com/inward/record.uri?eid=2-s2.0-

85083042384 \&doi=10.3390\%2fijerph17072359\&partner $I D=40 \& m d 5=d 6463 \mathrm{~b} 41$ aa 1752 ff $3710 \mathrm{c}$

$6455 c 795 d 71$

Hosseini, F.S., Choubin, B., Mosavi, A., Nabipour, N., Shamshirband, S., Darabi, H., Haghighi, A.T.

Flash-flood hazard assessment using ensembles and Bayesian-based machine learning models: Application of the simulated annealing feature selection method

(2020) Science of the Total Environment, 711, art. no. 135161, . https://www.scopus.com/inward/record.uri?eid=2-s2.0-

$85076251878 \& d o i=10.1016 \% 2 \mathrm{fj}$. scitotenv . $2019.135161 \&$ partnerI $D=40 \& \mathrm{md} 5=4 \mathrm{ff} 11428086 \mathrm{~d}$ $372298 \mathrm{ac} 4 \mathrm{e} 4181083 \mathrm{~b} 8 \mathrm{f}$

Asghar, M.Z., Subhan, F., Imran, M., Kundi, F.M., Khan, A., Shamshirband, S., Mosavi, A., Csiba, P., Varkonyi Koczy, A.R.

Performance evaluation of supervised machine learning techniques for efficient detection of emotions from online content

(2020) Computers, Materials and Continua, 63 (3), pp. 1093-1118.

https://www. scopus.com/inward/record.uri?eid=2-s2.0-

$85089755384 \&$ do $i=10.32604 \% 2$ fCMC .2020 .07709 \&partner $I D=40$ \&md5=230 ca81a $1 \mathrm{e} 1129 \mathrm{~d} 84 \mathrm{f} 59$

$78 \mathrm{~d} 8 \mathrm{a} 416 \mathrm{bd} 7 \mathrm{~d}$

Liu, Y., Abbasi, A.A., Aghaei, A., Abbasi, A., Mosavi, A., Shamshirband, S., Al-Qaness, M.A.A.

A Mobile Cloud-Based eHealth Scheme

(2020) Computers, Materials and Continua, 63 (1), pp. 31-39. 
https: / / www. scopus. com/inward/record.uri?eid=2-s2.085085598485 \&doi=10 . 32604\% $2 \mathrm{fcmc} .2020 .07708$ \&partner $\mathrm{D}=40$ \&md5=5ddf $1 \mathrm{c} 12 \mathrm{aelee} 89 \mathrm{~cd} 529$ $9 \mathrm{df} 8198663 \mathrm{f} 9$

Nosratabadi, S., Pinter, G., Mosavi, A., Semperger, S. Sustainable banking; Evaluation of the European business models (2020) Sustainability (Switzerland), 12 (6), art. no. 2314, . https: //www. scopus. com/inward/record.uri?eid=2-s2.0-

$85082809099 \&$ doi=10 . 3390\%2 fsul2 062314 \&partner $\mathrm{D}=40$ \&md5=f c $660 \mathrm{bb} 326 \mathrm{ab} 160478 \mathrm{~b} 7303 \mathrm{~b} 6$ $8 e 25260$

Harirchian, E., Lahmer, T., Buddhiraju, S., Mohammad, K., Mosavi, A. Earthquake safety assessment of buildings through rapid visual screening (2020) Buildings, 10 (3), art. no. 51, . https: //www. scopus. com/inward/record.uri?eid=2-s2.085082965226 \&doi=10.3390\%2 fbuildings 10030051 \&partner $\mathrm{D}=40$ \&md5=abeab0b34 $7 \mathrm{db} 462 \mathrm{a} 92$ 4 f2b51bae36057

Mousavi, S.N., Steinke, R., Jr., Teixeira, E.D., Bocchiola, D., Nabipour, N., Mosavi, A., Shamshirband, S.

Predictive modeling the free hydraulic jumps pressure through advanced statistical methods (2020) Mathematics, 8 (3), art. no. 323,. https: //www. scopus. com/inward/record.uri?eid=2-s2.0$85082421491 \&$ do $i=10.3390 \% 2$ math $8030323 \&$ partner $D=40$ \&md5=870 cfc $943 \mathrm{~b} 496267 \mathrm{bac} 808 \mathrm{a} 3$ $6419 \mathrm{ab} 82$

Dodangeh, E., Choubin, B., Eigdir, A.N., Nabipour, N., Panahi, M., Shamshirband, S., Mosavi, A.

Integrated machine learning methods with resampling algorithms for flood susceptibility prediction

(2020) Science of the Total Environment, 705, art. no. 135983,.

https://www. scopus. com/inward/record.uri?eid=2-s2.0-

$85076239035 \&$ doi=10 . $1016 \% 2 \mathrm{fj}$. scitotenv $.2019 .135983 \&$ partner $\mathrm{D}=40$ \&md5=b186a 8930659 1 c 2 c6f 96 e 4962213 e0e3

Saadatfar, H., Khosravi, S., Joloudari, J.H., Mosavi, A., Shamshirband, S. A new k-nearest neighbors classifier for big data based on efficient data pruning

(2020) Mathematics, 8 (2), art. no. 286,.

https: / / www. scopus. com/inward/record.uri?eid=2-s2.0-

$85080139012 \& \mathrm{do} i=10.3390 \% 2 \mathrm{fmath} 8020286$ \&partner $\mathrm{D}=40$ \&md5 =f c $8 \mathrm{a} 4 \mathrm{e} 66 \mathrm{~d} 2 \mathrm{bdb} 3 \mathrm{~d} 9 \mathrm{~b} 13193 \mathrm{e} 9$ ece 63574

Amirinasab, M., Shamshirband, S., Chronopoulos, A.T., Mosavi, A., Nabipour, N. Energy-efficient method for wireless sensor networks low-power radio operation in internet of things

(2020) Electronics (Switzerland), 9 (2), art. no. 320, .

https: / / www. scopus. com/inward/record.uri?eid=2-s2.0-

$85079516169 \& \mathrm{do} i=10.3390 \% 2$ felectronics 9020320 \&partner $\mathrm{D}=40$ \&md5=61b $7 \mathrm{~b} 215 \mathrm{cb} 79 \mathrm{e} 5 \mathrm{~d} 61$ c 9 e6d295d444801

Joloudari, J.H., Joloudari, E.H., Saadatfar, H., Ghasemigol, M., Razavi, S.M., Mosavi, A., Nabipour, N., Shamshirband, S., Nadai, L.

Coronary artery disease diagnosis; ranking the significant features using a random trees model

(2020) International Journal of Environmental Research and Public Health, 17

(3), art. no. 731, . 
https: / / www. scopus. com/inward/record.uri?eid=2-s2.0$85078236097 \&$ doi=10.3390\%2fijerph17030731\&partner $\mathrm{D}=40$ \&md5=a $7465703 \mathrm{c} 8 \mathrm{cac} 3 \mathrm{e} 7 \mathrm{c} 014 \mathrm{f}$ b 7 ae1c997c 9

Choubin, B., Abdolshahnejad, M., Moradi, E., Querol, X., Mosavi, A., Shamshirband, S., Ghamisi, P.

Spatial hazard assessment of the PM1O using machine learning models in Barcelona, Spain

(2020) Science of the Total Environment, 701, art. no. 134474, . https: / / www . scopus. com/inward/record.uri?eid=2-s2.085072669408 \&doi=10.1016\%2fj.scitotenv.2019.134474\&partner $\mathrm{dD}=40$ \&md5=d2b $7 \mathrm{a} 54 \mathrm{a} 03 \mathrm{a} 9$ d598e17d60334710f85d

Abbaspour-Gilandeh, Y., Molaee, A., Sabzi, S., Nabipur, N., Shamshirband, S., Mosavi, A.

A combined method of image processing and artificial neural network for the identification of 13 Iranian rice cultivars

(2020) Agronomy, 10 (1), art. no. 117,.

https: / / www . scopus. com/inward/record.uri ?eid=2-s2.0-

85077932476 \&doi=10 . 3390\%2 fagronomy1 $0010119 \&$ partner $\mathrm{D}=40$ \&md5=80 f20 c $3 \mathrm{cacb} 59$ e 30166 0 ebbc 867 c1 013

Khorampoor, N., Farahani, S.D., Mosavi, A.

Modeling the efficiency and emissions of a hybrid solar-gas power plant (2020) Engineering Applications of Computational Fluid Mechanics, 14 (1), pp. 790-804. https: / / www. scopus. com/inward/record.uri?eid=2-s2.0$85087382437 \&$ doi=10.1080\%2f19942060.2020.1778091\&partner $\mathrm{D}=40 \& \mathrm{md} 5=71 \mathrm{c} 3 \mathrm{~b} 724 \mathrm{ef} 4 \mathrm{e} 0 \mathrm{~d}$ 00 ealdb83f2a0a7f0f

Nosratabadi, S., Mosavi, A., Lakner, Z.

Food supply chain and business model innovation

(2020) Foods, 9 (2), art. no. 132, .

https: / / www. scopus. com/inward/record.uri ?eid=2-s2.0-

$85079173007 \&$ do $i=10.3390 \% 2$ f foods 9020132 \&partner $I D=40 \&$ md5=4ecc $3 a 77 \mathrm{c} 0 \mathrm{~d} 9142 \mathrm{db} 57063 \mathrm{c}$ $13 \mathrm{f} 9 \mathrm{~b} 406 \mathrm{~d}$

Ardabili, S., Mosavi, A., Várkonyi-Kóczy, A.R.

Building Energy Information: Demand and Consumption Prediction with Machine Learning Models for Sustainable and Smart Cities

(2020) Lecture Notes in Networks and Systems, 101, pp. 191-201.

https: / / www. scopus. com/inward/record.uri?eid=2-s2.0-

85078214612 \&doi=10.10072f978-3-030-36841-

8 _ $19 \&$ partner $I D=40$ \&md5=b 5 d 4 1b 6 a 5 b 37 f 1 bf 55 f 12 f 0 eaa 7989 b 4

Mosavi, A., Ardabili, S., Várkonyi-Kóczy, A.R.

List of Deep Learning Models

(2020) Lecture Notes in Networks and Systems, 101, pp. 202-214.

https: / / www. scopus. com/inward/record.uri ?eid=2-s2.0-

85078161946 \&do $i=10.1007 \div 2 f 978-3-030-36841-$

8_20\&partnerID $=40 \& \mathrm{md} 5=6553 \mathrm{ca} 74 \mathrm{abc} 48 \mathrm{a} 8 \mathrm{f} 6 \mathrm{ed} 8 \mathrm{fa} 11 \mathrm{a} 71 \mathrm{baf} 81$

Ardabili, S., Mosavi, A., Várkonyi-Kóczy, A.R.

Advances in Machine Learning Modeling Reviewing Hybrid and Ensemble Methods (2020) Lecture Notes in Networks and Systems, 101, pp. 215-227.

https: / / www . scopus. com/inward/record.uri ?eid=2-s2 . 0-

$85078132396 \& d o i=10.1007 \div 2 f 978-3-030-36841-$

$8 \_21 \&$ partner $I D=40 \&$ md5 =ce $8539 d 7$ fcca 6705 a 65 d 66668 a 9 d 4488 
Ardabili, S., Mosavi, A., Várkonyi-Kóczy, A.R. Systematic Review of Deep Learning and Machine Learning Models in Biofuels Research

(2020) Lecture Notes in Networks and Systems, 101, pp. 19-32.

https: / / www . scopus. com/inward/record.uri?eid=2-s2 . $0-$

$85078094140 \& d o i=10.1007 \circ 2 f 978-3-030-36841-$

8_2\&partnerID=40\&md5=7c619c339988d569f61d47f91466f491

Anwar, F., Qurat-Ul-Ain, Ejaz, M.Y., Mosavi, A.

A comparative analysis on diagnosis of diabetes mellitus using different approaches - A survey

(2020) Informatics in Medicine Unlocked, 21, art. no. 100482,.

https: / / www. scopus. com/inward/record.uri?eid=2-s2.0-

85097466304 \&doi=10.1016\%2fj.imu. 2020.100482 \&partnerID=4 0 \&md5=14c7ddd218a 52 e0049 cd7d1f24936190

Nosratabadi, S., Bahrami, P., Palouzian, K., Mosavi, A.

Leader cultural intelligence and organizational performance

(2020) Cogent Business and Management, 7 (1), art. no. 1809310, .

https: / / www. scopus. com/inward/record.uri?eid=2-s2.0-

85090085578 \&doi=10.1080\%2f23311975.2020.1809310\&partner $\mathrm{D}=40 \& \mathrm{md} 5=41 \mathrm{cb} 8 \mathrm{de} 85 \mathrm{eb} 030$

f475d513edd564a59f

Bemani, A., Baghban, A., Mosavi, A., S, S.

Estimating CO2-Brine diffusivity using hybrid models of ANFIS and evolutionary algorithms

(2020) Engineering Applications of Computational Fluid Mechanics, 14 (1), pp. 818-834.

https://www. scopus. com/inward/record.uri?eid=2-s2.0-

$85087523455 \&$ doi=10 . $1080 \% 2$ f19942060.2020.1774422\&partner ID=40\&md5=f0b $621 \mathrm{~b} 1905 \mathrm{a} 17$ 8132 ed33f215095d47

Khozani, Z.S., Sheikhi, S., Wan Mohtar, W.H.M., Mosavi, A. Forecasting shear stress parameters in rectangular channels using new soft computing methods

(2020) PLoS ONE, 15 (4), art. no. e0229731,.

https: / / www. scopus. com/inward/record.uri ?eid=2-s2.0-

85082999273 \&doi=10.13712 fjournal. pone. 0229731 \&partner $\mathrm{D}=40$ \&md5=0 f $90 \mathrm{f} 4 \mathrm{~d} 6 \mathrm{dl} \mathrm{adb} 4 \mathrm{~d}$ 5 ce9ea0669fa6a82b

Nabipour, N., Dehghani, M., Mosavi, A., Shamshirband, S. Short-Term Hydrological Drought Forecasting Based on Different Nature-Inspired Optimization Algorithms Hybridized with Artificial Neural Networks (2020) IEEE Access, 8, art. no. 8951168, pp. 15210-15222. https://www. scopus. com/inward/record.uri?eid=2-s2.085079217952 \&doi=10 . $1109 \% 2$ fACCESS . 2020.2964584 \&partner ID=40 \&md5=920adf6a 9 f 66 f 8 e 1 299556 c 52 b 57 cbod

Gundoshmian, T.M., Ardabili, S., Mosavi, A., Várkonyi-Kóczy, A.R. Prediction of Combine Harvester Performance Using Hybrid Machine Learning Modeling and Response Surface Methodology

(2020) Lecture Notes in Networks and Systems, 101, pp. 345-360. https: / / www. scopus. com/inward/record.uri?eid=2-s2.0$85078185202 \&$ doi=10.10072f $978-3-030-36841-$ 8_34\&partner $\mathrm{ID}=40$ \&md5=919ab42693b3e0078779fb412ae32d61

Ardabili, S., Mosavi, A., Dehghani, M., Várkonyi-Kóczy, A.R. 
Deep Learning and Machine Learning in Hydrological Processes Climate Change and Earth Systems a Systematic Review

(2020) Lecture Notes in Networks and Systems, 101, pp. 52-62.

https://www. scopus.com/inward/record.uri?eid=2-s2.0-

$85078077338 \& \mathrm{do} i=10.1007 \% 2 \mathrm{f} 978-3-030-36841-$

$8 \_5 \&$ partner ID $=40$ \&md5=28ab8a6e3257a 0 d8f 0090 ae 74 a $890 \mathrm{fe} 3$

Karami, M., Farahani, S.D., Kowsary, F., Mosavi, A.

Experimental estimation of temporal and spatial resolution of coefficient of heat transfer in a channel using inverse heat transfer method

(2020) Engineering Applications of Computational Fluid Mechanics, 14 (1), pp. 271-283.

https://www. scopus.com/inward/record.uri?eid=2-s2.0-

$85077675008 \&$ do $i=10.1080 \% 2 f 19942060.2020 .1712261$ \&partner $I D=40 \& \mathrm{md} 5=591$ ea $95 \mathrm{c} 76 \mathrm{e} 168$

6ee9a116951ed69265

Jabeen, T., Ashraf, H., Khatoon, A., Band, S.S., Mosavi, A.

A lightweight genetic based algorithm for data security in wireless body area networks

(2020) IEEE Access, 8, pp. 183460-183469.

https://www. scopus.com/inward/record.uri?eid=2-s2.0-

85102660631 \&do $i=10.1109 \% 2$ fACCESS .2020 .3028686 \&partner $I D=40$ \&md5=c19317d94475bbf 4

e9419ea8cac2a571

Band, S.S., Mohammadzadeh, A., Csiba, P., Mosavi, A., Varkonyi-Koczy, A.R. Voltage Regulation for Photovoltaics-Battery-Fuel Systems Using Adaptive Group Method of Data Handling Neural Networks (GMDH-NN)

(2020) IEEE Access, 8, art. no. 9253540, pp. 213748-213757.

https://www.scopus.com/inward/record.uri?eid=2-s2.0-

$85098055101 \&$ do $i=10.1109 \% 2$ fACCESS .2020 .3037134 \&partner $I D=40 \& \mathrm{md} 5=\mathrm{c} 24930 \mathrm{e} 486 \mathrm{~d} 23893$

$3 f 76 f c 7085 c 108 f c$

Mosavi, A., Hosseini, F.S., Choubin, B., Goodarzi, M., Dineva, A.A. Groundwater Salinity Susceptibility Mapping Using Classifier Ensemble and Bayesian Machine Learning Models

(2020) IEEE Access, 8, art. no. 9162111, pp. 145564-145576.

https://www. scopus.com/inward/record.uri?eid=2-s2.0-

85090277611 \&do $i=10.1109 \% 2$ fACCESS .2020 .3014908 \&partner $I D=40 \& \mathrm{md} 5=0543698 \mathrm{e} 8916 \mathrm{ff} 39$

b6aa03a5055b7ded

Nabipour, M., Nayyeri, P., Jabani, H., Shahab, S., Mosavi, A.

Predicting Stock Market Trends Using Machine Learning and Deep Learning

Algorithms Via Continuous and Binary Data; A Comparative Analysis

(2020) IEEE Access, 8, art. no. 9165760, pp. 150199-150212.

https://www.scopus.com/inward/record.uri?eid=2-s2.0-

$85090272444 \&$ do $i=10.1109 \% 2$ fACCESS .2020 .3015966 \&partner $I D=40 \& \mathrm{md} 5=329 \mathrm{f} 7 \mathrm{~b} 4$ a fedbea 58

$921 \mathrm{dc} 86 \mathrm{f} 8 \mathrm{e} 1248 \mathrm{ff}$

Shamshirband, S., Mosavi, A., Rabczuk, T., Nabipour, N., Chau, K.-W.

Prediction of significant wave height; comparison between nested grid numerical model, and machine learning models of artificial neural networks, extreme

learning and support vector machines

(2020) Engineering Applications of Computational Fluid Mechanics, 14 (1), pp. 805-817.

https: //www.scopus.com/inward/record.uri?eid=2-s2.0-

$85087401793 \& d o i=10.1080 \% 2 f 19942060.2020 .1773932 \&$ partner $I D=40 \& m d 5=336953 d 842 b 255$

$1 f 2 \mathrm{c} 1 \mathrm{f} 118560739830$ 
Pinter, G., Felde, I., Mosavi, A., Ghamisi, P., Gloaguen, R. COVID-19 pandemic prediction for Hungary; A hybrid machine learning approach (2020) Mathematics, 8 (6), art. no. 890,. https: //www. scopus. com/inward/record.uri?eid=2-s2.0-

$85086099997 \& d o i=10.3390 \% 2$ math 8060890 \&partnerID=40 \&md5=9d6 cf $0 \mathrm{~d} 64 \mathrm{eg} 9513 \mathrm{e} 70 \mathrm{c} 40 \mathrm{fd} 7$ $0955 \mathrm{ffdb}$

Nabipour, N., Mosavi, A., Baghban, A., Shamshirband, S., Felde, I. Extreme learning machine-based model for solubility estimation of hydrocarbon gases in electrolyte solutions (2020) Processes, 8 (1), art. no. 92,.

https: / / www. scopus. com/inward/record.uri?eid=2-s2.085079050611 \&do $i=10.3390 \% 2$ fpr 8010092 \&partner $I D=40$ \&md5=5a 1 e $8487 \mathrm{a} a 097 \mathrm{~b} 9 \mathrm{~b} 47 \mathrm{~d} 9 \mathrm{a} 82 \mathrm{e} 6 \mathrm{e}$ $8 \mathrm{e} 2 \mathrm{dc} 5$

Mohammadzadeh, D., Karballaeezadeh, N., Mohemmi, M., Mosavi, A., VárkonyiKóczy, A.R.

Urban Train Soil-Structure Interaction Modeling and Analysis

(2020) Lecture Notes in Networks and Systems, 101, pp. 361-381.

https: / / www. scopus. com/inward/record.uri?eid=2-s2.0-

$85078210468 \& d o i=10.1007 \circ 2$ f $978-3-030-36841-$

$8 \_35 \&$ partner $I D=40 \&$ md5 $=0$ b 51 bab 67 edb 2 c c $7 \mathrm{db} 2852$ d5 c 9 ee 2464

Nosratabadi, S., Mosavi, A., Keivani, R., Ardabili, S., Aram, F. State of the Art Survey of Deep Learning and Machine Learning Models for Smart Cities and Urban Sustainability (2020) Lecture Notes in Networks and Systems, 101 , pp. $228-238$.

https: / / www. scopus. com/inward/record.uri?eid=2-s2.0-

$85078134175 \& d o i=10.1007 \circ 2 f 978-3-030-36841-$

$8 \_22 \&$ partner $I D=40$ \&md5=eab2126d1b4997b6835e 9 e 480 b 0377 d 9

Mosavi, A., Salehi, F., Nadai, L., Karoly, S., Gorji, N.E. Modeling the temperature distribution during laser hardening process (2020) Results in Physics, 16, art. no. 102883, .

https://www. scopus. com/inward/record.uri?eid=2-s2.0-

85076865236 \&doi=10.1016\%2fj.rinp. 2019.102883 \&partner $\mathrm{D}=40$ \&md5=5aecb $66 \mathrm{de} 1721 \mathrm{a} 43 \mathrm{c}$ $58551 \mathrm{f} 012009 \mathrm{dd} 2$

Joloudari, J.H., Haderbadi, M., Mashmool, A., Ghasemigol, M., Band, S.S., Mosavi, A. Early detection of the advanced persistent threat attack using performance analysis of deep learning (2020) IEEE Access, 8, pp. 186125-186137. https: / / www . scopus. com/inward/record.uri?eid=2-s2 . 0-

85102795338 \&doi=10.1109\%2 fACCESS . 2020.3029202 \&partner $\mathrm{D}=40 \& \mathrm{md} 5=\mathrm{dbef} 9953 \mathrm{ccb} 73735$ a 51 f 856 a 526 b5 58 c

Ma, C., Mohammadzadeh, A., Turabieh, H., Mafarja, M., Band, S.S., Mosavi, A. Optimal Type-3 Fuzzy System for Solving Singular Multi-Pantograph Equations (2020) IEEE Access, 8, art. no. 9292666, pp. 225692-225702.

https: / / www. scopus. com/inward/record.uri?eid=2-s2.0-

$85098563867 \&$ doi=10 . $1109 \% 2$ fACCESS. 2020.3044548 \&partner $\mathrm{D}=40$ \&md5=ac36366 d4062a $9 \mathrm{~d} 6$ 6bbf78247c64fe93

Li, Y., Moradi, I., Kalantar, M., Babadi, E., Malekahmadi, O., Mosavi, A. Synthesis of new dihybrid nanofluid of TiO2/MWCNT in water-ethylene glycol to improve mixture thermal performance: preparation, characterization, and a novel correlation via ANN based on orthogonal distance regression algorithm (2020) Journal of Thermal Analysis and Calorimetry, . 
https://www. scopus.com/inward/record.uri?eid=2-s2.0$85096381085 \& d o i=10.1007 \% 2$ fs10973-020-10392-

9 \&partner I $D=40$ \&md5 $=4071178 \mathrm{ab} 77 \mathrm{~d} 2 \mathrm{ad} 7 \mathrm{dd} 6214 \mathrm{a} 656 \mathrm{cad} 057$

Mosavi, A., Golshan, M., Janizadeh, S., Choubin, B., Melesse, A.M., Dineva, A.A.

Ensemble models of GLM, FDA, MARS, and RF for flood and erosion susceptibility mapping: a priority assessment of sub-basins

(2020) Geocarto International, .

https: / / www . scopus. com/inward/record.uri?eid=2-s2.0-

$85093700283 \&$ doi=10 . $1080 \% 2$ f10106049.2020.1829101\&partner $\mathrm{D}=40$ \&md5=df $54 \mathrm{ba} 8 \mathrm{dd} 0 \mathrm{~b} 7 \mathrm{c} 5$

2409 fbe172e13bfef 4

Aram, F., Solgi, E., Baghaee, S., Higueras García, E., Mosavi, A., Band, S.S. How parks provide thermal comfort perception in the metropolitan cores; a case study in Madrid Mediterranean climatic zone (2020) Climate Risk Management, 30, art. no. 100245,.

https: / / www . scopus. com/inward/record.uri?eid=2-s2.0-

85091627976 \&doi=10.1016\%2fj.crm. $2020.100245 \&$ partner $\mathrm{dD}=40$ \&md5=50bca 060 f $84899389 \mathrm{a}$ 24 ebbb 9 f 464642

Madvar, H.R., Dehghani, M., Memarzadeh, R., Salwana, E., Mosavi, A., Shamshirband, S. Derivation of optimized equations for estimation of dispersion coefficient in natural streams using hybridized ANN with PSO and CSO Algorithms (2020) IEEE Access, 8, art. no. 9177117, pp. 156582-156599.

https: / / www . scopus. com/inward/record.uri?eid=2-s2.0-

85091248674 \&doi=10.1109\%2 fACCESS. 2020.3019362 \&partner $\mathrm{D}=40$ \&md5=2176 d249d760edc 8 ee47c9eae9a69e13

Abbasi, A.A., Shamshirband, S., Al-Qaness, M.A.A., Abbasi, A., Al-Jallad, N.T., Mosavi, A. Resource-aware network topology management framework

(2020) Acta Polytechnica Hungarica, 17 (4), pp. 89-101.

https: / / www. scopus. com/inward/record.uri?eid=2-s2.0-

85090671588 \&doi=10 . 12700\%2 fAPH . 17.4.2020.4 . 5 \&partner $\mathrm{D}=40$ \&md5=9b800417191353055 $16 \mathrm{e} 4 \mathrm{f} 49 \mathrm{~d} 162 \mathrm{fb} 44$

Rahman, A., Nasir, M.K., Rahman, Z., Mosavi, A., Shahab, S., Minaei-Bidgoli, B. DistBlockBuilding: A Distributed Blockchain-Based SDN-IoT Network for Smart Building Management (2020) IEEE Access, 8, art. no. 9151145, pp. 140008-140018. https: / / www . scopus. com/inward/record.uri?eid=2-s2.0-

$85089530642 \&$ do $i=10.1109 \% 2$ fACCESS $.2020 .3012435 \&$ partner $\mathrm{D}=40 \& \mathrm{md} 5=302 \mathrm{ad} 2742 \mathrm{ab} 41188$ 35 dcecdba6ba233a

Nabipour, N., Mosavi, A., Hajnal, E., Nadai, L., Shamshirband, S., Chau, K.-W. Modeling climate change impact on wind power resources using adaptive neurofuzzy inference system (2020) Engineering Applications of Computational Fluid Mechanics, 14 (1), pp. 491-506. https: / / www . scopus. com/inward/record.uri?eid=2-s2 . 0 $85079824247 \&$ doi=10.1080\%2f19942060.2020.1722241\&partnerID=40\&md5=30a9afe80 c7a $9 \mathrm{f}$ $1 \mathrm{fb} 495 \mathrm{fe} 7 \mathrm{f} 7 \mathrm{be} 2 \mathrm{bdfa}$

Shamshirband, S., Joloudari, J.J., GhasemiGol, M., Saadatfar, H., Mosavi, A., Nabipour, N. FCS-MBFLEACH: Designing an energy-aware fault detection system for mobile wireless sensor networks (2020) Mathematics, 8 (1), art. no. 28 , . https: / / www. scopus. com/inward/record.uri?eid=2-s2.0$85079614293 \&$ doi=10.3390\%2 fMATH $8010028 \&$ partnerID=4 0 \&md5=f 4 be $1 \mathrm{cfe} 34 \mathrm{cdd} 4434 \mathrm{e} 048 \mathrm{a} 8 \mathrm{C}$ 57158491 
Homaei, M.H., Soleimani, F., Shamshirband, S., Mosavi, A., Nabipour, N., Varkonyi-Koczy, A.R. An Enhanced Distributed Congestion Control Method for Classical 6LowPAN Protocols Using Fuzzy Decision System (2020) IEEE Access, 8 , art. no. 8967114, pp. 20628-20645.

https: / / www. scopus. com/inward/record.uri?eid=2-s2.0-

$85079536026 \&$ doi=10 . $1109 \% 2$ fACCESS. $2020.2968524 \&$ partner $\mathrm{D}=40 \& \mathrm{md} 5=16937217 \mathrm{a} 68 \mathrm{~cd} 993$ $6 a 4 d 27 d 0 a 1 f 4 e 286$

Dehghani, M., Salehi, S., Mosavi, A., Nabipour, N., Shamshirband, S., Ghamisi, P. Spatial analysis of seasonal precipitation over Iran: Co-variation with climate indices (2020) ISPRS International Journal of Geo-Information, 9 (2), art. no. 9020073,.

https: / / www. scopus. com/inward/record.uri?eid=2-s2.085078407642 \&doi=10.3390\%2fijgi 9020073 \&partnerID=4 0 \&md5=3e $8 \mathrm{~d} 5 \mathrm{f} 413 \mathrm{e} 86 \mathrm{a} 03 \mathrm{fb} 4 \mathrm{df} 3 \mathrm{ab} 1$ 332524 a 0

Ardabili, S., Mosavi, A., Mahmoudi, A., Gundoshmian, T.M., Nosratabadi, S., Várkonyi-Kóczy, A.R. Modelling Temperature Variation of Mushroom Growing Hall Using Artificial Neural Networks (2020) Lecture Notes in Networks and Systems, 101 , pp. 33-45.

https://www. scopus. com/inward/record.uri ?eid=2-s2.0-

$85078166598 \& d o i=10.1007 \div 2$ f $978-3-030-36841-$

$8 \_3 \&$ partnerID $=40$ \&md5=2044fff0a651a128693550d748339427

Wang, Z., Fathollahzadeh Attar, N., Khalili, K., Behmanesh, J., Band, S.S., Mosavi, A., Chau, K.-W. Monthly streamflow prediction using a hybrid stochastic-deterministic approach for parsimonious non-linear time series modeling (2020) Engineering Applications of Computational Fluid Mechanics, 14 (1), pp. 1351-1372.

https: / / www . scopus. com/inward/record.uri ?eid=2-s2 . 0 -

$85093834545 \&$ doi=10.1080\%2f19942060.2020.1830858\&partner $\mathrm{D}=40 \& \mathrm{md} 5=151 \mathrm{c} 9 \mathrm{f} 283 \mathrm{f} 045 \mathrm{e}$ $10544 \mathrm{~b} 142891052 \mathrm{ec} 2$

Karballaeezadeh, N., Zaremotekhases, F., Shamshirband, S., Mosavi, A., Nabipour, N., Csiba, P., Várkonyi-Kóczy, A.R. Intelligent road inspection with advanced machine learning; Hybrid prediction models for smart mobility and transportation maintenance systems (2020) Energies, 13 (7), art. no. en13071718, .

https://www. scopus. com/inward/record.uri?eid=2-s2.0-

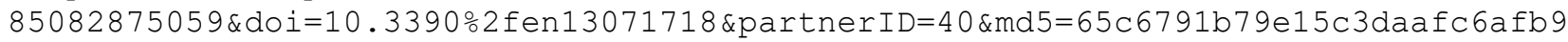
fa 9 bcf 9

Kargar, K., Samadianfard, S., Parsa, J., Nabipour, N., Shamshirband, S., Mosavi, A., Chau, K.-W. Estimating longitudinal dispersion coefficient in natural streams using empirical models and machine learning algorithms (2020) Engineering Applications of Computational Fluid Mechanics, 14 (1), pp. $311-322$. https: / / www . scopus. com/inward/record.uri ?eid=2-s2 . 0 85079169372 \&doi=10.1080\%2f19942060.2020.1712260\&partnerID=40\&md5=7ef3e14dee $7 \mathrm{f} 0 \mathrm{~b}$ $19 e 487468 b 9 f 95715 e$

Shamshirband, S., Babanezhad, M., Mosavi, A., Nabipour, N., Hajnal, E., Nadai, L., Chau, K.-W. Prediction of flow characteristics in the bubble column reactor by the artificial pheromone-based communication of biological ants (2020) Engineering Applications of Computational Fluid Mechanics, 14 (1), pp. $367-378$. 
https: / / www. scopus. com/inward/record.uri?eid=2-s2.0$85079144246 \&$ doi=10 . 1080\%2f19942060.2020.1715842\&partner ID=40\&md5=1b13246a 90 e 853 $52 \mathrm{~d} 4$ eae 5 eaceb50cb5

Shabani, S., Samadianfard, S., Sattari, M.T., Mosavi, A., Shamshirband, S., Kmet, T., Várkonyi-Kóczy, A.R. Modeling pan evaporation using Gaussian Process Regression K-Nearest Neighbors Random Forest and support vector machines; comparative analysis (2020) Atmosphere, 11 (1), art. no. 66, . https: / / www . scopus. com/inward/record.uri?eid=2-s2 . 0$85079071027 \&$ do $i=10.3390 \% 2$ fATMOS1 1010066 \&partner $\mathrm{D}=40$ \&md5=1d $6551 \mathrm{bf5a} 58 \mathrm{bef} 100764 \mathrm{~d}$ 07 a $95059 d 5$

Faroughi, M., Karimimoshaver, M., Aram, F., Solgi, E., Mosavi, A., Nabipour, N., Chau, K.-W. Computational modeling of land surface temperature using remote sensing data to investigate the spatial arrangement of buildings and energy consumption relationship (2020) Engineering Applications of Computational Fluid Mechanics, 14 (1), pp. 254-270.

https: / / www . scopus. com/inward/record.uri?eid=2-s2 . 0-

$85077290098 \&$ doi=10.1080\%2f19942060.2019.1707711\&partner $\mathrm{D}=40 \& \mathrm{md} 5=\mathrm{a} 0 \mathrm{c} 1 \mathrm{~d} 987 \mathrm{e} 494 \mathrm{fc}$ b425e5flc51aa4550a

Hemmati-Sarapardeh, A., Hajirezaie, S., Soltanian, M.R., Mosavi, A., Nabipour, N., Shamshirband, S., Chau, K.-W. Modeling natural gas compressibility factor using a hybrid group method of data handling (2020) Engineering Applications of Computational Fluid Mechanics, 14 (1), pp. 27-37. https: / / www. scopus. com/inward/record.uri ?eid=2-s2.085074897544 \&doi=10.1080\%2f19942060.2019.1679668\&partner $\mathrm{D}=40 \& \mathrm{md} 5=367785243 \mathrm{~d} 627 \mathrm{~d}$ f446f561982 be13eb 9

Ardabili, S.F., Mosavi, A., Ghamisi, P., Ferdinand, F., Varkonyi-Koczy, A.R., Reuter, U., Rabczuk, T., Atkinson, P.M. COVID-19 outbreak prediction with machine learning (2020) Algorithms, 13 (10), art. no. 249, . https: / / www. scopus. com/inward/record.uri?eid=2-s2.0-

$85099545833 \&$ doi=10 $.3390 \% 2 \mathrm{fa} 13100249$ \&partnerID=40\&md5=5f $6 \mathrm{c} 128 \mathrm{a} 695 \mathrm{cf} 86 \mathrm{c} 2 \mathrm{~d} 113 \mathrm{fe} 265$ e 92540

Ahmadi, M.H., Baghban, A., Sadeghzadeh, M., Zamen, M., Mosavi, A., shamshirband, S., Kumar, R., Mohammadi-Khanaposhtani, M. Evaluation of electrical efficiency of photovoltaic thermal solar collector (2020) Engineering Applications of Computational Fluid Mechanics, 14 (1), pp. 545-565. https: / / www. scopus. com/inward/record.uri ?eid=2-s2 . 0 85080051117 \&doi=10.1080\%2f19942060.2020.1734094\&partner $\mathrm{D}=40 \& \mathrm{md} 5=38091 \mathrm{~b} 1 \mathrm{fc} 06 \mathrm{a} 41$ $76 \mathrm{ead} 725 \mathrm{a} 5 \mathrm{fc} 4 \mathrm{cbd} 6 \mathrm{~b}$

Mosavi, A., Shirzadi, A., Choubin, B., Taromideh, F., Hosseini, F.S., Borji, M., Shahabi, H., Salvati, A., Dineva, A.A. Towards an Ensemble Machine Learning Model of Random Subspace Based Functional Tree Classifier for Snow Avalanche Susceptibility Mapping (2020) IEEE Access, 8, art. no. 9160950, pp. 145968145983 .

https: / / www . scopus. com/inward/record.uri?eid=2-s2 . 0$85090275342 \&$ doi=10.1109\%2fACCESS . $2020.3014816 \&$ partner $\mathrm{D}=40 \& \mathrm{md} 5=01 \mathrm{fdbd} 16 \mathrm{~d} 08 \mathrm{c} 48 \mathrm{ba}$ 894420 bce08829b6

Shamshirband, S., Esmaeilbeiki, F., Zarehaghi, D., Neyshabouri, M., Samadianfard, S., Ghorbani, M.A., Mosavi, A., Nabipour, N., Chau, K.-W. Comparative analysis of hybrid models of firefly optimization algorithm with support vector machines and multilayer perceptron for predicting soil 
temperature at different depths (2020) Engineering Applications of computational Fluid Mechanics, 14 (1), pp. 939-953.

https: / / www. scopus. com/inward/record.uri ?eid=2-s2.0-

$85087827960 \&$ doi=10.1080\%2f19942060.2020.1788644\&partner ID=4 0 \&d5=439802e $7 \mathrm{ad} 2471$ ba65aeaca0491647d

Asadi, E., Isazadeh, M., Samadianfard, S., Ramli, M.F., Mosavi, A., Nabipour, N., Shamshirband, S., Hajnal, E., Chau, K.-W. Groundwater quality assessment for sustainable drinking and irrigation (2020) Sustainability (Switzerland), 12 (1), art. no. 177, .

https: / / www . scopus. com/inward/record.uri?eid=2-s2.0-

$85079389649 \&$ doi=10.3390\%2fsu12010177 \&partnerID=40\&md5=0 $6 \mathrm{a} 50 \mathrm{f} 45 \mathrm{c} 67 \mathrm{~d} 72002 \mathrm{~b} 344 \mathrm{e} 61 \mathrm{f}$ c5248f6

Ouaer, H., Hosseini, A.H., Amar, M.N., Seghier, M.E.A.B., Ghriga, M.A., Nabipour, N., Andersen, P.O., Mosavi, A., Shamshirband, S. Rigorous connectionist models to predict carbon dioxide solubility in various ionic liquids (2020) Applied Sciences (Switzerland), 10 (1), art. no. 304, . https: / / www . scopus. com/inward/record.uri?eid=2-s2.0-

$85079044757 \&$ doi=10 . 3390\%2 fapp1 0010304 \&partnerID=4 0 \&md5=e 6a 54 d3fa 1889 dc 961 a 73 ef 0 f1f4560f

Shamshirband, S., Hashemi, S., Salimi, H., Samadianfard, S., Asadi, E., Shadkani, S., Kargar, K., Mosavi, A., Nabipour, N., Chau, K.-W. Predicting Standardized Streamflow index for hydrological drought using machine learning models (2020) Engineering Applications of Computational Fluid Mechanics, 14 (1), pp. 339-350.

https://www. scopus. com/inward/record.uri?eid=2-s2.0-

$85079229630 \& d o i=10.1080 \% 2 f 19942060.2020 .1715844 \& p a r t n e r I D=40 \& m d 5=e f e 54552531 d b 2$ c1a4c28b8a4857e7e7

Nabipour, N., Karballaeezadeh, N., Dineva, A., Mosavi, A., Mohammadzadeh S., D., Shamshirband, S. Comparative analysis of machine learning models for prediction of remaining service life of flexible pavement (2019) Mathematics, 7 (12), art. no. 1198,.

https: / / www. scopus. com/inward/record.uri ?eid=2-s2.0-

85079617248 \&doi=10 . 3390\%2 fMATH 7121198 \&partner $\mathrm{D}=40$ \&md5=81bca $341 \mathrm{~d} 1 \mathrm{f} 1 \mathrm{f} 5 \mathrm{eeb} 9999821$

$7 \mathrm{c} 1845 \mathrm{fc}$

Dineva, A., Mosavi, A., Gyimesi, M., Vajda, I., Nabipour, N., Rabczuk, T. Fault diagnosis of rotating electrical machines using multi-label lassification (2019) Applied Sciences (Switzerland), 9 (23), art. no. 5086, . https: / / www. scopus. com/inward/record.uri?eid=2-s2.0-

$85077005423 \&$ doi=10 . 3390\%2 fapp9235086 \&artner ID=4 0 \&md5=b 47 f 66 a 332 f 4 e 4 aaa 04 be 5141 $980 a 30 f$

Choubin, B., Mosavi, A., Alamdarloo, E.H., Hosseini, F.S., Shamshirband, S., Dashtekian, K., Ghamisi, P. Earth fissure hazard prediction using machine learning models (2019) Environmental Research, 179, art. no. 108770, . https: //www. scopus. com/inward/record.uri?eid=2-s2.0-

85072674450 \&doi=10.1016\%2fj. envres. 2019.108770 \&partner $\mathrm{D}=40$ \&md5=93 dbb51 cbe 95426 $2 \mathrm{bf3c0c6a1a3c104b}$

Mansoor, K., Ghani, A., Chaudhry, S.A., Shamshirband, S., Ghayyur, S.A.K., Mosavi, A.

Securing IoT-based RFID systems: A robust authentication protocol using symmetric cryptography

(2019) Sensors (Switzerland), 19 (21), art. no. 4752, . 
https: / / www. scopus. com/inward/record.uri?eid=2-s2.0-

85074549911 \&doi=10 . 3390\%2fs19214752\&partner ID=40\&md5=711932946402edec $640 \mathrm{~d} 890655$ a 9 a 1 b2

Choubin, B., Heydari Alamdarloo, E., Mosavi, A., Sajedi Hosseini, F., Ahmad, S., Goodarzi, M., Shamshirband, S.

Spatiotemporal dynamics assessment of snow cover to infer snowline elevation mobility in the mountainous regions

(2019) Cold Regions Science and Technology, 167, art. no. 102870, .

https://www. scopus. com/inward/record.uri?eid=2-s2.0-

$85071257983 \&$ doi=10.1016\%2fj. coldregions. 2019.102870 \&partner $\mathrm{dD}=40 \mathrm{kmd5}=6 \mathrm{c} 6 \mathrm{c} 9 \mathrm{fa} 098$ a 42 eef 9 fd66730dffed 2 d7

Abedinnezhad, S., Ahmadi, M.H., Pourkiaei, S.M., Pourfayaz, F., Mosavi, A., Feidt, M., Shamshirband, S.

Thermodynamic assessment and multi-objective optimization of performance of irreversible dual-miller cycle (2019) Energies, 12 (20), art. no. 4000, .

https: //www. scopus. com/inward/record.uri ?eid=2-s2.0-

85075031500 \&doi=10 . 3390\%2 fen 12204000 \&partner $\mathrm{D}=40$ \&md5=31b4dd6a 8 deba 57 a $9 \mathrm{~b} 7952242$ bd77397

Aram, F., Solgi, E., García, E.H., Mosavi, A., Várkonyi-Kóczy, A.R. The cooling effect of large-scale urban parks on surrounding area thermal comfort

(2019) Energies, 12 (20), art. no. 3904, . https: / / www. scopus. com/inward/record.uri ?eid=2-s2.0-

85074990704 \&doi=10.3390\%2 fen 12203904 \&partner $I D=40$ \&md5=776738b5a8598f7078806fdbc 8 C55799

Shamshirband, S., Hadipoor, M., Baghban, A., Mosavi, A., Bukor, J., VárkonyiKóczy, A.R.

Developing an ANFIS-PSO model to predict mercury emissions in combustion flue gases

(2019) Mathematics, 7 (10), art. no. 965,.

https: / / www. scopus. com/inward/record.uri?eid=2-s2.0-

$85073780684 \&$ do $i=10.3390 \% 2$ fmath 7100965 \&partner $\mathrm{D}=40$ \&md5=4 faa $335511 \mathrm{~d} 9 \mathrm{~b} 93 \mathrm{a} 5473 \mathrm{~d} 0 \mathrm{e} 2$

$03 \mathrm{bc} 62 \mathrm{da}$

Choubin, B., Borji, M., Mosavi, A., Sajedi-Hosseini, F., Singh, V.P., Shamshirband, S.

Snow avalanche hazard prediction using machine learning methods

(2019) Journal of Hydrology, 577, art. no. 123929, .

https: / / www. scopus. com/inward/record.uri?eid=2-s2.0-

$85068524113 \& d o i=10.1016 \% 2 \mathrm{fj} \cdot j$ hydrol . $2019.123929 \&$ partner $\mathrm{D}=40$ \&md5=fb5d5277 c5bf32 $5 \mathrm{~d} 77 \mathrm{bc} 2 \mathrm{e} 9 \mathrm{eea} 96 \mathrm{ea} 3 \mathrm{c}$

Samadianfard, S., Jarhan, S., Salwana, E., Mosavi, A., Shamshirband, S., Akib, S.

Support vector regression integrated with fruit fly optimization algorithm for river flow forecasting in lake urmia basin

(2019) Water (Switzerland), 11 (9), art. no. 1934,. https: //www. scopus. com/inward/record.uri ?eid=2-s2.0-

85072559131 \&doi=10.3390\%2 fw1 091934 \&partner $I D=40$ \&md5=df $98 \mathrm{a} 2362 \mathrm{~d} 63 \mathrm{e} 41141 \mathrm{c} 2516 \mathrm{eb} 6$ $65 c 521$

Perez, H., Tah, J.H.M., Mosavi, A. 
Deep learning for detecting building defects using convolutional neural networks

(2019) Sensors (Switzerland), 19 (16), art. no. 3556, .

https://www. scopus.com/inward/record.uri?eid=2-s2.0-

85071457857 \&do $i=10.3390 \% 2$ fs 19163556 \&partner $I D=40$ \&md5=0 c5bce 6 af 99227 b 0b1 65074 ea 1 8be 7 a 9

Danial Mohammadzadeh, S., Kazemi, S.-F., Mosavi, A., Nasseralshariati, E., Tah, J.H.M.

Prediction of compression index of fine-grained soils using a gene expression programming model

(2019) Infrastructures, 4 (2), art. no. infrastructures4020026, .

https://www.scopus.com/inward/record.uri?eid=2-s2.0-

$85073368454 \&$ do $i=10.3390 \% 2$ finfrastructures 4020026 \&partner ID $=40$ \&md5=4 c1b 62 f $013 f 95$ $6 e 2 e 7 a 77 b 17073 f c 3 b 6$

Dineva, A., Mosavi, A., Ardabili, S., Vajda, I., Shamshirband, S., Rabczuk, T., Chau, K. $-W$.

Review of soft computing models in design and control of rotating electrical machines

(2019) Energies, 12 (6), art. no. 1049, .

https://www. scopus.com/inward/record.uri?eid=2-s2.0-

$85065339282 \&$ do $i=10.3390 \% 2$ fen 12061049 \&partner $I D=40 \&$ md $5=4434$ b 84020 dd 4 e 43 ecbcd 4 af $9 \mathrm{c} 7 \mathrm{c} 52 \mathrm{~d}$

Qasem, S.N., Samadianfard, S., Nahand, H.S., Mosavi, A., Shamshirband, S., Chau, K.-W. Estimating daily dew point temperature using machine learning algorithms (2019) Water (Switzerland), 11 (3), art. no. 582, . https://www. scopus.com/inward/record.uri?eid=2-s2.0$85065021146 \&$ do $i=10.3390 \% 2 \mathrm{fw} 11030582$ \&partner I $=40$ \&md5=79ff172375f 7877928816 deba 7 a $21 \mathrm{f} 2 \mathrm{c}$

Choubin, B., Moradi, E., Golshan, M., Adamowski, J., Sajedi-Hosseini, F., Mosavi, A. An ensemble prediction of flood susceptibility using multivariate discriminant analysis, classification and regression trees, and support vector machines (2019) Science of the Total Environment, 651, pp. 2087-2096. https: / /www. scopus.com/inward/record.uri?eid=2-s2.0$85054727882 \& d o i=10.1016 \% 2 \mathrm{fj}$. scitotenv $.2018 .10 .064 \&$ partnerI $\mathrm{D}=40$ \&md5=7b158984eea 0 $3 \mathrm{~b} 3 \mathrm{c} 21178 \mathrm{f} 1973219446$

Dehghani, M., Riahi-Madvar, H., Hooshyaripor, F., Mosavi, A., Shamshirband, S., Zavadskas, E.K., Chau, K.-W. Prediction of hydropower generation using Grey wolf optimization adaptive neuro-fuzzy inference system

(2019) Energies, 12 (2), art. no. 289, .

https://www. scopus.com/inward/record.uri?eid=2-s2.0-

$85060517286 \&$ do $i=10.3390 \% 2$ fen 12020289 \&partner $I D=40$ \&md5=1bd374 c 7 a 9986809 e 817 bbf 78 $5766 f 88$

Rezakazemi, M., Mosavi, A., Shirazian, S. ANFIS pattern for molecular membranes separation optimization (2019) Journal of Molecular Liquids, 274, pp. 470-476. https: / /www. scopus.com/inward/record.uri?eid=2-s2.0-

$85056243905 \& \mathrm{do} i=10.1016 \% 2 \mathrm{fj} . \mathrm{molliq} .2018 .11 .017$ \&partner $\mathrm{D}=40$ \&md5=6b 4 ef5ba $8971 \mathrm{~d} 83$ $72 \mathrm{a} 07 \mathrm{bc} 7 \mathrm{e} 19 \mathrm{cbc} 234$

Mosavi, A., Edalatifar, M. A Hybrid Neuro-Fuzzy Algorithm for Prediction of Reference Evapotranspiration (2019) Lecture Notes in Networks and Systems, 53, pp. $235-243$. 
https://www.scopus.com/inward/record.uri?eid=2-s2.0-

$85055675657 \&$ doi $=10.1007 \% 2 f 978-3-319-99834-$

331 \&partner $I D=40 \& m d 5=f 907$ fa $63 b 73794$ cbbca $99 f 2$ f $2 d c 65474$

Khansari, N.M., Farrokhi, A., Mosavi, A. Orthotropic mode II shear test

fixture: Iosipesque modification (2019) Engineering Solid Mechanics, 7 (2), pp. 93-108.

https: / / www . scopus. com/inward/record.uri?eid=2-s2.0-

$85065191092 \&$ doi=10 . 5267\%2fj.esm. $2019.4 .003 \&$ partner $\mathrm{I}=40$ \&md5=ec $3 \mathrm{~d} 4193 \mathrm{be} 476887373$ 392866 c 979323

Ghalandari, M., Shamshirband, S., Mosavi, A., Chau, K.-W. Flutter speed estimation using presented differential quadrature method formulation (2019) Engineering Applications of Computational Fluid Mechanics, 13 (1), pp. $804-810$. https: / / www. scopus. com/inward/record.uri?eid=2-s2.0$85069968273 \&$ do $i=10.1080 \% 2$ f19942060.2019.1627676 \&partner $\mathrm{D}=40 \& \mathrm{md} 5=6 \mathrm{~d} 5 \mathrm{abbc1} 1 \mathrm{acd} 0 \mathrm{e}$ $00 \mathrm{bd} 3481 \mathrm{df} 59185 \mathrm{ae} 5$

Zhang, S., Karimi, S., Shamshirband, S., Mosavi, A. Optimization algorithm for reduction the size of Dixon resultant matrix: A case study on mechanical application (2019) Computers, Materials and Continua, 58 (2), pp. 567-583. https: / / www. scopus. com/inward/record.uri?eid=2-s2.0-

$85064824055 \&$ do $i=10.32604 \% 2 \mathrm{fcmc} .2019 .02795 \&$ partner $\mathrm{ID}=40$ \&md5=26264a $57 \mathrm{cee} 40682 \mathrm{~d} 0 \mathrm{c} 4$ d0 0438 c 878 c 7 b

Mosavi, A., Shamshirband, S., Salwana, E., Chau, K.-W., Tah, J.H.M. Prediction of multi-inputs bubble column reactor using a novel hybrid model of computational fluid dynamics and machine learning (2019) Engineering Applications of Computational Fluid Mechanics, 13 (1), pp. 482-492. https: //www. scopus. com/inward/record.uri?eid=2-s2.0$85069537510 \&$ doi=10.10802 f19942060.2019.1613448\&partner $\mathrm{D}=40 \& \mathrm{md} 5=5984 \mathrm{a} 0 \mathrm{e} 27 \mathrm{c} 5840$ 49 de lea2b9f6b073d2

Ghalandari, M., Bornassi, S., Shamshirband, S., Mosavi, A., Chau, K.W. Investigation of submerged structures' flexibility on sloshing frequency using a boundary element method and finite element analysis (2019) Engineering Applications of Computational Fluid Mechanics, 13 (1), pp. 519-528. https: / / www. scopus. com/inward/record.uri?eid=2-s2.0$85069528021 \&$ doi=10.1080\%2f19942060.2019.1619197\&partner $\mathrm{D}=40 \& \mathrm{md} 5=\mathrm{d} 5 \mathrm{c} 51 \mathrm{dca} 922 \mathrm{f} 4 \mathrm{f}$ $2 e 7 f 6$ bf $043 a$ ec 22659

Torabi, M., Hashemi, S., Saybani, M.R., Shamshirband, S., Mosavi, A. A Hybrid clustering and classification technique for forecasting short-term energy consumption (2019) Environmental Progress and Sustainable Energy, 38 (1), pp. 66-76. https: / / www. scopus. com/inward/record.uri ?eid=2-s2.0$85059887500 \&$ doi=10 . $1002 \% 2 \mathrm{fep} .12934 \&$ partner $\mathrm{D}=40$ \&md5=8517b $6536 \mathrm{f} 6 \mathrm{aa} 606 \mathrm{~d} 001 \mathrm{e} 88 \mathrm{db} 69$ 77945

Torabi, M., Mosavi, A., Ozturk, P., Varkonyi-Koczy, A., Istvan, V. A Hybrid Machine Learning Approach for Daily Prediction of Solar Radiation (2019) Lecture Notes in Networks and Systems, 53, pp. 266-274. https: / / www. scopus. com/inward/record.uri?eid=2-s2.0$85055710201 \& d o i=10.1007 \% 2 f 978-3-319-99834-$ 3_35\&partner ID $=40$ \&md5=32dfbf 237 f0d 49 f $96106 \mathrm{~b} 36 f 51332287$ 
Jafari-Sejahrood, A., Najafi, B., Faizollahzadeh Ardabili, S., Shamshirband, S., Mosavi, A., Chau, K.-W. Limiting factors for biogas production from cow manure: energo-environmental approach (2019) Engineering Applications of Computational Fluid Mechanics, 13 (1), pp. 954-966. https: //www. scopus.com/inward/record.uri?eid=2-s2.0$85071971160 \&$ do $i=10.1080 \% 2 f 19942060.2019 .1654411$ \&partner $I D=40 \& m d 5=$ fafef 2 cafbe 582 fe 4 b 8 eab54638b918e

Ghalandari, M., Ziamolki, A., Mosavi, A., Shamshirband, S., Chau, K.-W., Bornassi, S. Aeromechanical optimization of first row compressor test stand blades using a hybrid machine learning model of genetic algorithm, artificial neural networks and design of experiments (2019) Engineering Applications of Computational Fluid Mechanics, 13 (1), pp. 892-904. https: / / www. scopus. com/inward/record.uri?eid=2-s2.0$85071049579 \&$ do $i=10.1080 \% 2$ f19942060 . $2019.1649196 \&$ partner $\mathrm{D}=40 \& \mathrm{md} 5=\mathrm{b} 2367 \mathrm{f} 7944 \mathrm{cdb} 8$ $0 d 328 b 50420 a 76510 d$

Menad, N.A., Noureddine, Z., Hemmati-Sarapardeh, A., Shamshirband, S., Mosavi, A., Chau, K.-W. Modeling temperature dependency of oil - water relative permeability in thermal enhanced oil recovery processes using group method of data handling and gene expression programming (2019) Engineering Applications of Computational Fluid Mechanics, 13 (1), pp. 724-743. https: / / www. scopus. com/inward/record.uri?eid=2-s2.0$85069765019 \&$ do $i=10.1080 \% 2$ f19942060.2019.1639549\&partner $\mathrm{D}=40$ \&md5=58aa 0 a 2 fa 3 f6b5 b0e965e25e2a9f8f 5 f

Aram, F., Solgi, E., García, E.H., Mohammadzadeh, S.D., Mosavi, A., Shamshirband, S. Design and validation of a computational program for analysing mental maps: Aram mental map analyzer (2019) Sustainability (Switzerland), 11 (14), art. no. 3790, . https: / / www. scopus. com/inward/record.uri?eid=2-s2.0-

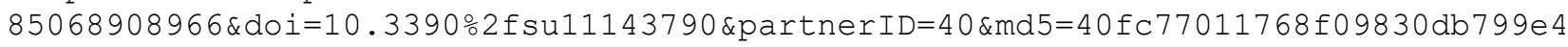
22 be 696

Karballaeezadeh, N., Mohammadzadeh S, D., Shamshirband, S., Hajikhodaverdikhan, P., Mosavi, A., Chau, K.-W. Prediction of remaining service life of pavement using an optimized support vector machine (case study of Semnan-Firuzkuh road) (2019) Engineering Applications of Computational Fluid Mechanics, 13 (1), pp. $188-198$.

https: / / www. scopus. com/inward/record.uri ?eid=2-s2.0-

85065872178 \&doi=10.1080\%2f19942060.2018.1563829\&partner $\mathrm{dD}=40$ \&md5=707 c $7620 \mathrm{dfd} 08 \mathrm{~d}$ fb2789ea1f7e43b207

Mosavi, A., Salimi, M., Ardabili, S.F., Rabczuk, T., Shamshirband, S., Varkonyi-Koczy, A.R. State of the art of machine learning models in energy systems, a systematic review (2019) Energies, 12 (7), art. no. 1301, . https: //www. scopus. com/inward/record.uri?eid=2-s2.0-

85065016714 \&do $i=10.3390 \% 2$ fen12071301 \&partner $I D=40$ \&md5=4a 12 e $6969 \mathrm{~d} 000 \mathrm{c} 09 \mathrm{f} 96 \mathrm{~b} 650 \mathrm{a} 1$ $15 \mathrm{~b} 65 \mathrm{bb}$

Nosratabadi, S., Mosavi, A., Shamshirband, S., Zavadskas, E.K., Rakotonirainy, A., Chau, K.W. Sustainable business models: A review (2019) Sustainability (Switzerland), 11 (6), art. no. 1663,. https: / / www. scopus. com/inward/record.uri ?eid=2-s2.085063466318 \&doi=10.3390\%2f sul1 061663 \&partner $I D=40$ \&md5=165318a9355c7c70be $9263 \mathrm{dce}$ $68 \mathrm{a} 73 \mathrm{ae}$ 
Shamshirband, S., Jafari Nodoushan, E., Adolf, J.E., Abdul Manaf, A., Mosavi, A., Chau, K.-W. Ensemble models with uncertainty analysis for multi-day ahead forecasting of chlorophyll a concentration in coastal waters

(2019) Engineering Applications of Computational Fluid Mechanics, 13 (1), pp. 91-101.

https: / / www. scopus. com/inward/record.uri?eid=2-s2.0-

$85060552327 \&$ do $i=10.1080 \% 2$ f19942060 . 2018.1553742 \&partner $\mathrm{D}=40$ \&md5=bc $9113 \mathrm{eac} 4 \mathrm{aafd}$ $43 \mathrm{~b} 1355 \mathrm{a} 047306186 \mathrm{~b}$

Riahi-Madvar, H., Dehghani, M., Seifi, A., Salwana, E., Shamshirband, S., Mosavi, A., Chau, K.-W. Comparative analysis of soft computing techniques RBF, MLP, and ANFIS with MLR and MNLR for predicting grade-control scour hole geometry (2019) Engineering Applications of Computational Fluid Mechanics, 13 (1), pp. 529-550. https: / / www. scopus. com/inward/record.uri?eid=2-s2.0$85069530236 \&$ do $i=10.1080 \% 2 \mathrm{f} 19942060.2019 .1618396 \&$ partner $\mathrm{D}=40 \& \mathrm{md} 5=7 \mathrm{f} 74949571 \mathrm{edd} 4$ d8baaa 0 abb $99303 e 18$

Farzaneh-Gord, M., Faramarzi, M., Ahmadi, M.H., Sadi, M., Shamshirband, S., Mosavi, A., Chau, K.-W. Numerical simulation of pressure pulsation effects of a snubber in a CNG station for increasing measurement accuracy (2019) Engineering Applications of Computational Fluid Mechanics, 13 (1), pp. 642-663. https: / / www. scopus. com/inward/record.uri?eid=2-s2.085069524578 \&doi=10.1080\%2f19942060.2019.1624197\&partner ID=40\&md5=cb6a597 cf $94 \mathrm{bc} 2$ f36320e7ec4la7c46f

Ardabili, S.F., Najafi, B., Alizamir, M., Mosavi, A., Shamshirband, S., Rabczuk, T. Using SVM-RSM and ELM-RSM approaches for optimizing the production process of methyl and ethyl esters (2018) Energies, 11 (11), art. no. 2889, . https://www. scopus.com/inward/record.uri?eid=2-s2.0-

85055690958 \&doi=10 . 3390\%2 fen 11112889 \&partner $I D=40$ \&md5=cd 41 f $6 \mathrm{bc} 98394 \mathrm{aa} 96 \mathrm{~d} 9 \mathrm{ddb} 001$ b17da 79

Mosavi, A., Ozturk, P., Chau, K.-W. Flood prediction using machine learning models: Literature review (2018) Water (Switzerland), 10 (11), art. no. 1536, . https: / / www. scopus. com/inward/record.uri?eid=2-s2.0-

$85055710433 \& d o i=10.3390 \% 2 \mathrm{fw} 10111536$ \&partner $\mathrm{ID}=40$ \&md5=88678d4e $73 \mathrm{f} 95350 \mathrm{f} 266299 \mathrm{fb} 0$ 582669

Moeini, I., Ahmadpour, M., Mosavi, A., Alharbi, N., Gorji, N.E. Modeling the detection efficiency in photodetectors with temperature-dependent mobility and carrier lifetime (2018) Superlattices and Microstructures, 122, pp. 557-562. https: / / www. scopus. com/inward/record.uri?eid=2-s2.0-

85049500060 \&doi=10.1016\%2fj.spmi.2018.06.052 \&partner $\mathrm{D}=40$ \&md5=d0e61 a $921 \mathrm{~d} 50 \mathrm{~d} 1780$ c02c502764a5474

Darvishzadeh, A., Alharbi, N., Mosavi, A., Gorji, N.E. Modeling the strain impact on refractive index and optical transmission rate (2018) Physica B: Condensed Matter, 543, pp. 14-17.

https: / / www. scopus. com/inward/record.uri ?eid=2-s2.085047016448 \&doi=10.1016\%2fj.physb. $2018.05 .001 \&$ partner $\mathrm{d}=40 \mathrm{\& md5}=\mathrm{e} 5 \mathrm{cc} 4692 \mathrm{f} 10 \mathrm{f} 87 \mathrm{~d} 3$ 6 celeaba533a810e

Moeini, I., Ahmadpour, M., Mosavi, A., Alharbi, N., Gorji, N.E. Modeling the time-dependent characteristics of perovskite solar cells (2018) Solar Energy, 170 , pp. 969-973. 
https: / / www. scopus. com/inward/record.uri?eid=2-s2.0$85048548859 \& d o i=10.1016 \% 2 \mathrm{fj}$. solener.2018.05.082 \&partner ID=40\&md5=6fa $8 \mathrm{~d} 38396 \mathrm{eff} 8$ 812 dfade 5760 e13a 48

Imani, M.H., Zalzar, S., Mosavi, A., Shamshirband, S. Strategic Behavior of Retailers for Risk Reduction and Profit Increment via Distributed Generators and Demand Response Programs (2018) Energies, 11 (6), art. no. 1602, . https: / / www . scopus. com/inward/record.uri?eid=2-s2.0-

$85049385993 \&$ do $i=10.3390 \% 2$ fen $11061602 \&$ partner $I D=40 \& \mathrm{md} 5=\mathrm{f} \mathrm{cdb} 4663 f 74 \mathrm{cb} 4882 \mathrm{~d} 053940 \mathrm{~d}$ $27 \mathrm{c} 880 \mathrm{~d}$

Najafi, B., Ardabili, S.F., Mosavi, A., Shamshirband, S., Rabczuk, T. An intelligent artificial neural network-response surface methodology method for accessing the optimum biodiesel and diesel fuel blending conditions in a diesel engine from the viewpoint of exergy and energy analysis (2018) Energies, 11 (4), art. no. 860, . https: / / www. scopus. com/inward/record.uri?eid=2-s2.0$85045297280 \& d o i=10.3390 \% 2$ fen 11040860 \&partner $I D=40$ \&md5=3f1360bbba $9 c 3415331992845$ 7 a 64 ado

Mosavi, A., Benkreif, R., Varkonyi-Koczy, A.R. Comparison of Euler-Bernoulli and Timoshenko beam equations for railway system dynamics (2018) Advances in Intelligent Systems and Computing, 660, pp. 32-40. https: //www. scopus. com/inward/record.uri?eid=2-s2.0$85029820098 \& d o i=10.1007 \div 2$ f $978-3-319-67459-$

9_5\&partnerID $=40 \& \mathrm{md} 5=96039 \mathrm{ef} 150448 \mathrm{cf} 01 \mathrm{~cd} 81 \mathrm{a} 031 \mathrm{~b} 2 \mathrm{ecfa} 5$

Mosavi, A., Rabczuk, T., Varkonyi-Koczy, A.R. Reviewing the novel machine learning tools for materials design (2018) Advances in Intelligent Systems and Computing, 660, pp. 50-58. https: //www. scopus. com/inward/record.uri?eid=2-s2.0$85029812280 \& d o i=10.1007 \div 2$ f $978-3-319-67459-$ $9 \_7 \&$ partner $I D=40 \& \mathrm{md} 5=4 \mathrm{~b} 306 \mathrm{~d} 6 \mathrm{cc} 865 \mathrm{c} 882 \mathrm{ea} 7 \mathrm{ed} 484 \mathrm{cefcc} 014$

Mosavi, A., Rituraj, R., Varkonyi-Koczy, A.R. Review on the usage of the multiobjective optimization package of modeFrontier in the energy sector (2018) Advances in Intelligent systems and Computing, 660, pp. 217-224. https: / / www. scopus. com/inward/record.uri?eid=2-s2.0$85029807217 \& d o i=10.1007 \div 2 f 978-3-319-67459-$

9_28\&partnerID $=40 \& \mathrm{md} 5=8 \mathrm{e} 087 \mathrm{e} 52258 \mathrm{de} 63450112836784600 \mathrm{df}$

Mosavi, A., Lopez, A., Varkonyi-Koczy, A.R. Industrial applications of big data: State of the art survey (2018) Advances in Intelligent Systems and Computing, 660, pp. 225-232.

https: / / www . scopus. com/inward/record.uri?eid=2-s2 . 0$85029803270 \&$ do $i=10.1007 \div 2$ f $978-3-319-67459-$

9_29\&partnerID $=40$ \&md5=8fa 440 a 7 d1896e 8 f2c376fe 466 albblc

Mosavi, A., Bathla, Y., Varkonyi-Koczy, A. Predicting the future using web knowledge: State of the art survey (2018) Advances in Intelligent Systems and Computing, 660, pp. 341-349. https: / / www . scopus. com/inward/record.uri?eid=2-s2.0$85029799290 \&$ doi=10.1007\%2f978-3-319-67459$9 \_42 \&$ partner $I D=40$ \&md5=36d0224 c895b338f 1 a 8820 dfa $95695 \mathrm{~d} 1$

Mousavi, S., Mosavi, A., Varkonyi-Koczy, A.R. A load balancing algorithm for resource allocation in cloud computing (2018) Advances in Intelligent Systems and Computing, 660, pp. 289-296. 
https://www.scopus.com/inward/record.uri?eid=2-s2.0-

$85029796640 \&$ doi=10.1007\%2f978-3-319-67459-

9_36\&partner $I D=40 \& m d 5=e d a 892$ f2535e5e681eadf 03 dcfaeced 6

Baranyai, M., Mosavi, A., Vajda, I., Varkonyi-Koczy, A.R.

Optimal design of electrical machines: State of the art survey

(2018) Advances in Intelligent Systems and Computing, 660, pp. 209-216.

https://www. scopus. com/inward/record.uri?eid=2-s2.0-

$85029820224 \& d o i=10.1007 \div 2 f 978-3-319-67459-$

$9 \_27 \&$ partner $I D=40 \& \mathrm{md} 5=37 \mathrm{f} 7 \mathrm{fc} 6553 \mathrm{c} 2 \mathrm{c} 7 \mathrm{e} 8 \mathrm{a} 445 \mathrm{c} 0 \mathrm{cb} 6 \mathrm{fea} 231$

Maghsoodi, A.I., Maghsoodi, A.I., Mosavi, A., Rabczuk, T., Zavadskas, E.K. Renewable energy technology selection problem using integrated H-SWARA-

MULTIMOORA approach (2018) Sustainability (Switzerland), 10 (12), art. no.

4481 , .

https: / / www . scopus. com/inward/record.uri?eid=2-s2.0-

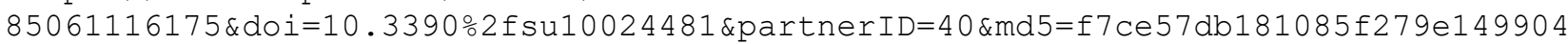
2180373

Fardad, K., Najafi, B., Ardabili, S.F., Mosavi, A., Shamshirband, S., Rabczuk, T. Biodegradation of medicinal plants waste in an anaerobic digestion reactor for biogas production (2018) Computers, Materials and Continua, 55 (3), pp. 318-392.

https: / / www . scopus. com/inward/record.uri?eid=2-s2.0-

85049172058 \&do $i=10.3970 \% 2 \mathrm{fmc} .2018 .01803$ \&partner $\mathrm{D}=40$ \&md5=a $9239302 \mathrm{abbb} 3 \mathrm{f} 5 \mathrm{~b} 74 \mathrm{e} 61$ ebec282a $8 \mathrm{~d} 0$

Taherei Ghazvinei, P., Darvishi, H.H., Mosavi, A., Bin Wan Yusof, K., Alizamir, M., Shamshirband, S., Chau, K.-W. Sugarcane growth prediction based on meteorological parameters using extreme learning machine and artificial neural network (2018) Engineering Applications of Computational Fluid Mechanics, 12 (1), pp. 738-749. https://www. scopus. com/inward/record.uri?eid=2-s2.0$85055686653 \&$ doi=10 . $1080 \% 2$ f19942060.2018.1526119\&partner $\mathrm{D}=40$ \&md5=0 dd $676 \mathrm{df} 14 \mathrm{fb} 05$ 9c61173c0117f01cf9 